Hydroécol. Appl. (1991) 1, pp. 63 - 110

\title{
Impact thermique des effluents du Centre de Production Nucléaire du Bugey sur les invertébrés benthiques du Rhône
}

\author{
M.C. Roger, B. Faessel, M. Lafont \\ CEMAGREF \\ Division Biologie des Ecosystèmes Aquatiques, \\ Laboratoire d'Ecologie des Invertébrés Aquatiques, 3, quai Chauveau - 69336 Lyon Cedex 09
}

Résumé. - Les rejets d'eau chaude du Centre de Production Nucléaire du Bugey entraînent jusqu'à $5 \mathrm{~km}$ en aval de la centrale, une élévation de la température des eaux du Rhône de $6^{\circ} \mathrm{C}$ en moyenne. L'échauffement des eaux est encore sensible au niveau de la confluence avec l'Ain.

Suite à l'étude d'impact réalisée en 1975 dans le cadre de la mise en service de ce centre, une réactualisation des données s'avérait nécessaire sur le site pour effectuer un bilan de l'impact réel des rejets thermiques sur les biocénoses.

L'examen de la faune benthique met en évidence une diminution notable de la richesse taxonomique sur l'ensemble du site entre 1975 et 1988-89. Ainsi, Rhithrogena diaphana, Ecdyonurus sp. (éphéméroptères) et les représentants de la famille des Unionidae (mollusques) sont absents en 1988, la densité des oligochètes a diminué depuis 1975 sur le point amont.

Au niveau de la station échauffée, on observe en 1988-89 une réduction significative de la richesse taxonomique des invertébrés, avec notamment la disparition de Potamanthus luteus (éphéméroptère) et de Propappus volki (oligochète). On constate également une diminution de la densité des Chironomidae et des oligochètes, ces derniers, ainsi que les Gammaridae, disparaissant durant la période la plus chaude (août, septembre).

Mots clés : Fleuve, invertébrés benthiques, pollution thermique.

Summary. - The Bugey Nuclear Power plant induces a $6{ }^{\circ} \mathrm{C}$ mean rise of temperature of the Rhone river water. The temperature rise is perceptible up to $10 \mathrm{~km}$ downstream the power plant.

Invertebrates were collected during 1975 when the power plant still had not reached its full functioning. A new study was carried out during 1988-89 to update these early data, what was necessary to assess the impact of a temperature rise on benthic invertebrates.

The taxonomic richness of invertebrate communities showed a decrease since 1975 on the whole investigated area. The ephemeroptera Rhithrogena diaphana, and Ecdyonurus sp. 
the Unionidae (Mollusca) are absent from 1988-89 surveys. The oligochaete abundance decreases in the upstream station when compared to the 1975 field investigations. Pollution seems to be responsible for the observed changes in the structure of benthic invertebrate communities.

When compared with the upstream site, the warmed station is characterized by a significant reduction of the taxonomic richness of invertebrate communities, by the eradication of such species as Potamanthus luteus (Ephemeroptera) and Propappus volki (Oligochaeta), by a reduction of Chironomidae and Oligochaeta abundances and by the disparition of Oligochaeta and Gammaridae during the warmer season (August, September). A synergy between pollution and temperature rises is suggested as an explanation for these observations.

Keys words: River, benthic invertebrates, thermal pollution.

\section{INTRODUCTION - PROBLÉMATIQUE}

L'implantation de la centrale du Bugey sur le Haut-Rhône a donné lieu entre 1975 et 1977 à une étude d'impact sur les plans physico-chimique et hydrobiologique. Cette étude consistait d'une part en une description de l'état du Rhône à l'époque, d'autre part en une évaluation des conséquences du fonctionnement de la centrale vis-à-vis de la faune benthique et des peuplements piscicoles. Ce travail a été mené en s'appuyant sur la détermination des organismes au niveau spécifique, tant en ce qui concerne les poissons que les invertébrés benthiques. Les prévisions ont donc été établies en terme de changements dans la composition des peuplements.

A partir de 1978 , les suivis physico-chimiques et hydrobiologiques ont permis de vérifier la validité des prévisions faites en ce qui concerne les peuplements de poissons, et de préciser les perturbations induites par le réchauffement artificiel des eaux. L'influence de la centrale a également été évaluées sur la faune benthique en utilisant des méthodes globales d'interprétation, sans détermination au niveau spécifique.

La synthèse de ces données pose certaines questions, dont les principales sont:

- Quelle est la situation actuelle de ce secteur? A-t-il été le siège de modifications perceptibles depuis les années 1975, en particulier en ce qui concerne la qualité du milieu ?

- Qu'en est-il de l'influence du réchauffement artificiel des eaux sur les peuplements d'invertébrés?

Après un rappel de l'évolution physico-chimique du site, l'étude concernera l'ensemble des espèces ou taxons de macroinvertébrés benthiques. La prise en considération des espèces d'oligochètes fera cependant l'objet d'un chapitre particulier, compte tenu de l'utilisation, pour ce groupe d'invertébrés, de protocoles spécifiques de traitement des échantillons. 


\section{1. - PRÉSENTATION DU MILIEU}

\subsection{Les centrales thermiques}

Le Centre de Production Nucléaire du Bugey est situé à $35 \mathrm{~km}$ à l'est de Lyon sur la rive droite du Rhône en amont de la confluence avec l'Ain (fig. 1). II comporte 5 tranches dont les caractéristiques sont notées dans le tableau 1.

\subsection{Matériels et méthodes}

Le problème qui se posait résidait dans la possibilité de disposer de relevés comparables entre 1975 et 1987-88-89.

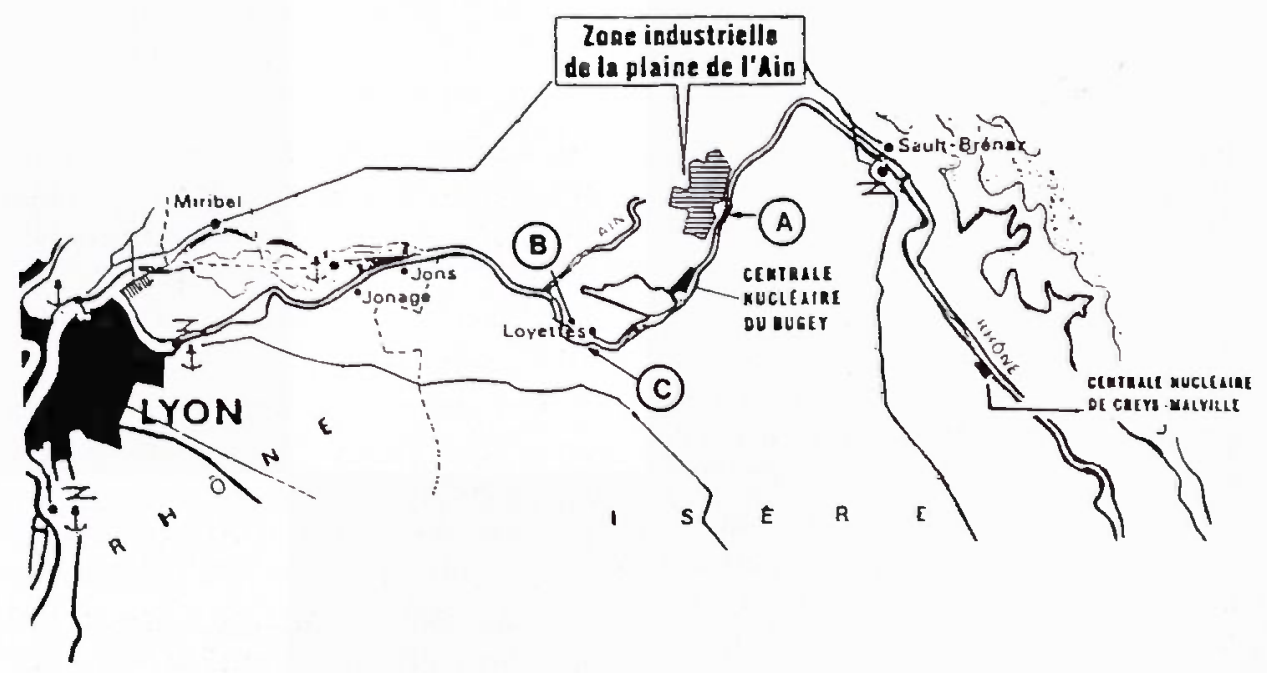

Fig. 1. - Situation des points de prélèvement.

Fig. 1. - Location of the sampling stations.

Tableau 1. - Caractéristiques des tranches nucléaires implantées sur le site du Bugey. Table 1. - Présentation of the Bugey Nuclear Power Plant.

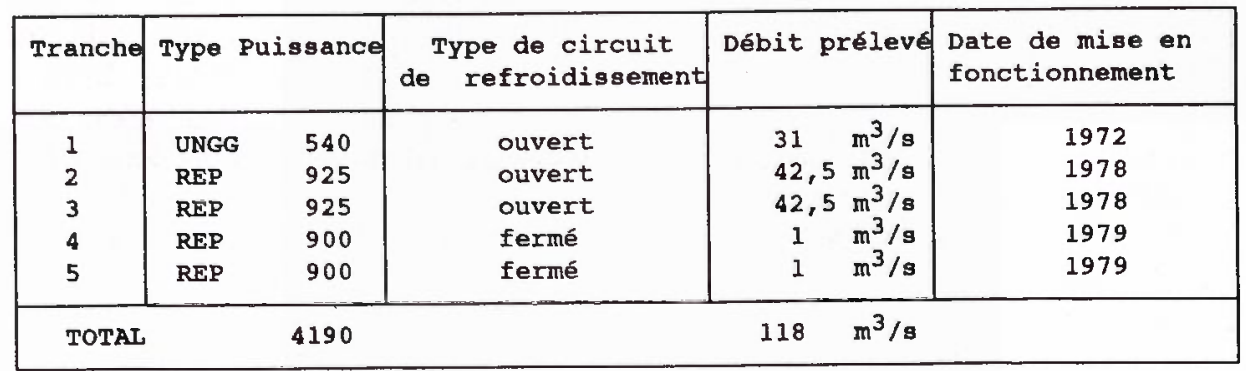


L'état de référence a pour base d'une part le rapport CTGREF (1977) et d'autre part le travail de Perrin (1978).

Dans l'étude CTGREF, cinq campagnes de prélèvements ont été effectuées en 1975 (tableau 2) sur le site de St Vulbas (St A., fig. 1) en rive droite essentiellement. L'échantillonnage a été réalisé suivant le protocole préconisé dans la méthode des IQBG (Verneaux et al. 1976) (six prélèvements par station, en tenant compte du couple vitesse du courant-substratum).

Dans la thèse de Perrin, seules ont été retenues les campagnes effectuées en 1976 (tableau 1) sur le site de St Vulbas (St A.), les prélèvements étant réalisés sur la rive gauche. Cet auteur a récolté la faune à l'aide des substrats artificiels décrits par Roux et al. 1976.

L'état actuel du site du Bugey a été étudié à partir de prélèvements de faune benthique effectués en 1988-

Tableau 2. - Dates et modes de prélèvements.

Table 2. - Sampling methodology and sampling seasons.

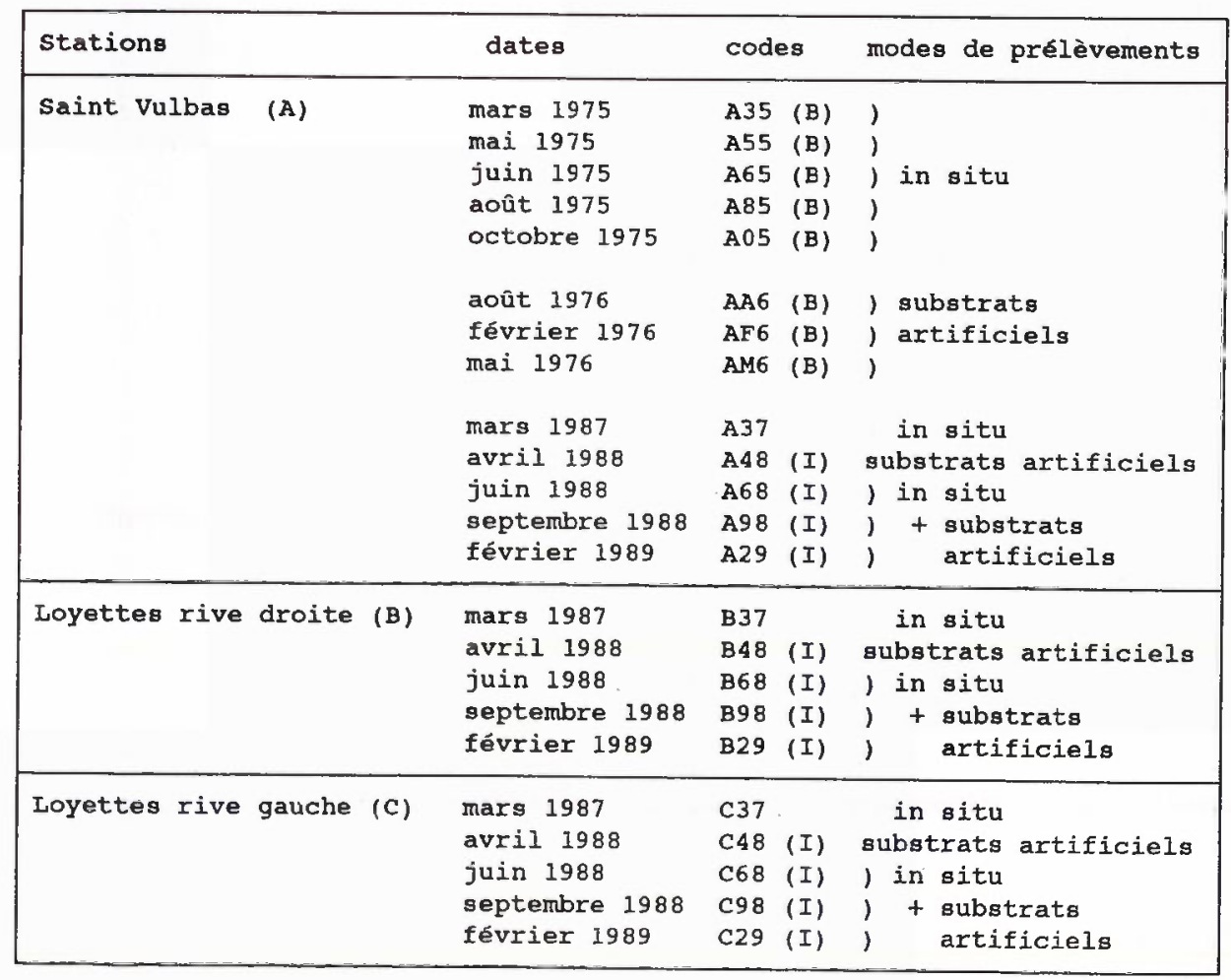


1989 (tableau 2) suivant le protocole IQBG et IQBP : échantillonnage in situ et substrats artificiels de type CEMAGREF (Verneaux et al. 1976).

Dans la mesure où les oligochètes avaient été examinés en 1975 à partir de prélèvements réalisés selon ce protocole, la même procédure a été conservée par la suite. II n'était donc pas souhaitable, dans le cadre précis de cette étude, de se conformer strictement au protocole d'échantillonnage préconisé spécifiquement pour les oligochètes (Lafont, 1989). En revanche le tamisage des échantillons récents a été effectué, au laboratoire, sur un filtre de $0,315 \mathrm{~mm}$ de vide de maille (vide de maille utilisé en 1975). De plus, les sédiments fins étant actuellement inexistants sur le site (à l'inverse des années 1975), seuls les sédiments grossiers ont été pris ici en considération.

Trois stations ont été retenues, leur emplacement est établi en fonction du rejet thermique (fig. 1).

La station $A$ (cf. station $A$, CTGREF-Perrin) est localisée à $4 \mathrm{~km}$ environ en amont de la centrale. La température de l'eau varie de $5,2^{\circ} \mathrm{C}$ en hiver à $23,5^{\circ} \mathrm{C}$ en été (fig. 2).

Les stations $B$ et $C$ sont situées en aval, à environ $5 \mathrm{~km}$ de la centrale :

- en rive droite la station $B$ est fortement influencée par le réchauffement (fig. 2). Les valeurs extrêmes de température sur cette station sont de $8,3^{\circ} \mathrm{C}$ en février-mars et $29,8^{\circ} \mathrm{C}$ en août. L'élévation de température est sensible jusqu'à la confluence de l'Ain.
- en rive gauche la station $C$ est soumise à une élévation de température plus faible. En moyenne les températures de cette station sont supérieures de $0,6^{\circ} \mathrm{C}$ à celles de la station de référence (valeur calculée d'après 116 mesures ponctuelles relevées entre 1978 et 1989).

Les différentes dates et les modes de prélèvements ainsi que les codes des stations sont indiqués dans le tableau 2.

Parallèlement à l'échantillonnage de la faune benthique, des prélèvements d'eau ont été effectués en 1975 et en 1988-89 afin d'analyser les principaux paramètres physico-chimiques permettant une comparaison de la qualité de l'eau entre ces deux périodes.

L'année d'étude (1988-89) est caractérisée par un débit moyen de $560 \mathrm{~m}^{3} / \mathrm{s}$, des crues relativement importantes en janvier, mars et surtout en octobre où le débit atteint 1700 $\mathrm{m}^{3} / \mathrm{s}$ (fig. 3). L'étiage a eu lieu en septembre, octobre avec un débit inférieur à $200 \mathrm{~m}^{3} / \mathrm{s}$.

\section{2. - PHYSICO-CHIMIE}

\section{1. État actuel}

14 paramètres physico-chimiques ont été retenus pour apprécier la qualité du support aqueux (tab. 3 et 4).

- $\mathrm{Ph}$

Le $\mathrm{Ph}$ mesuré sur le secteur est légèrement basique avec des valeurs 


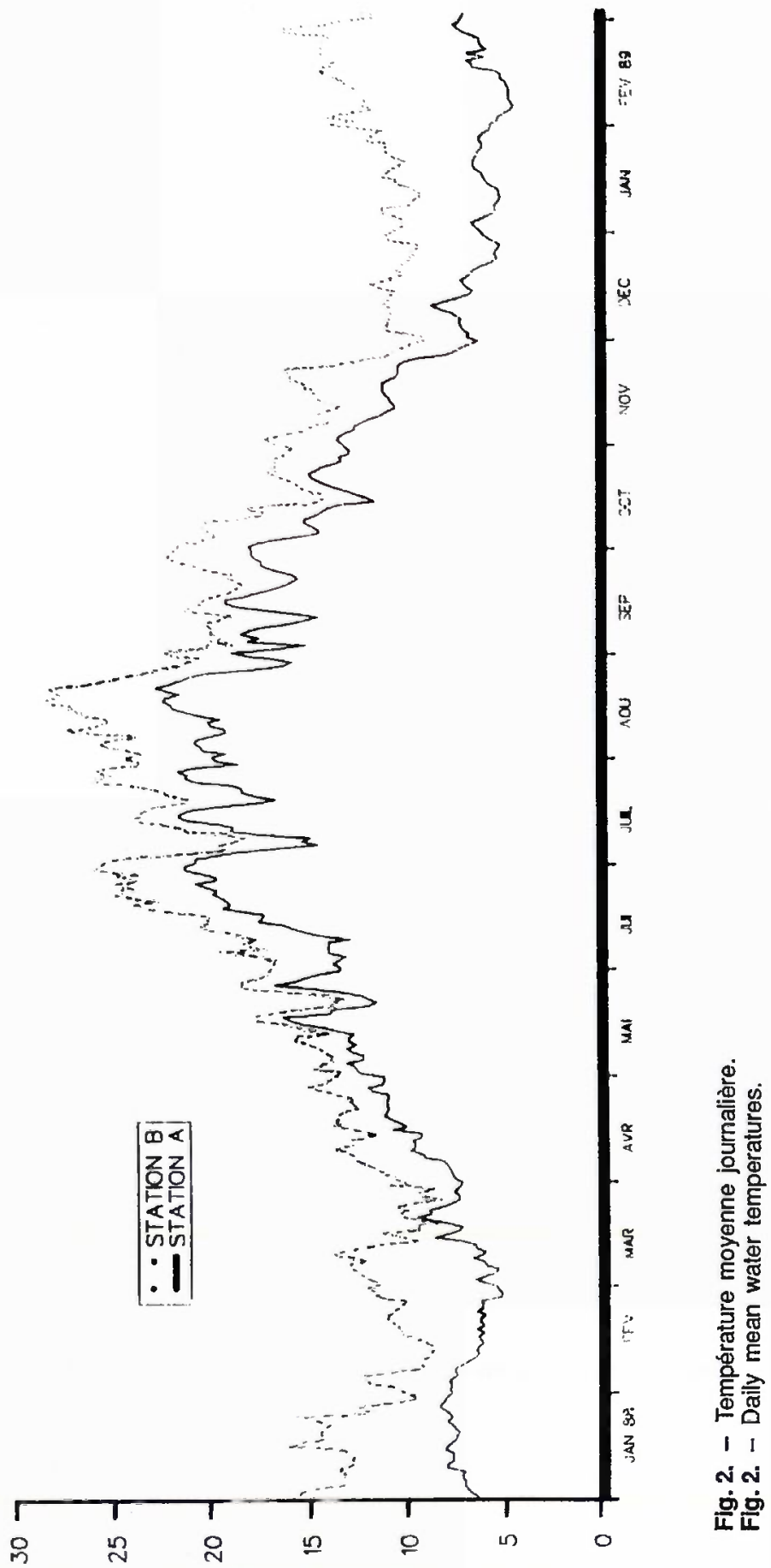

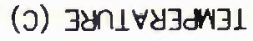




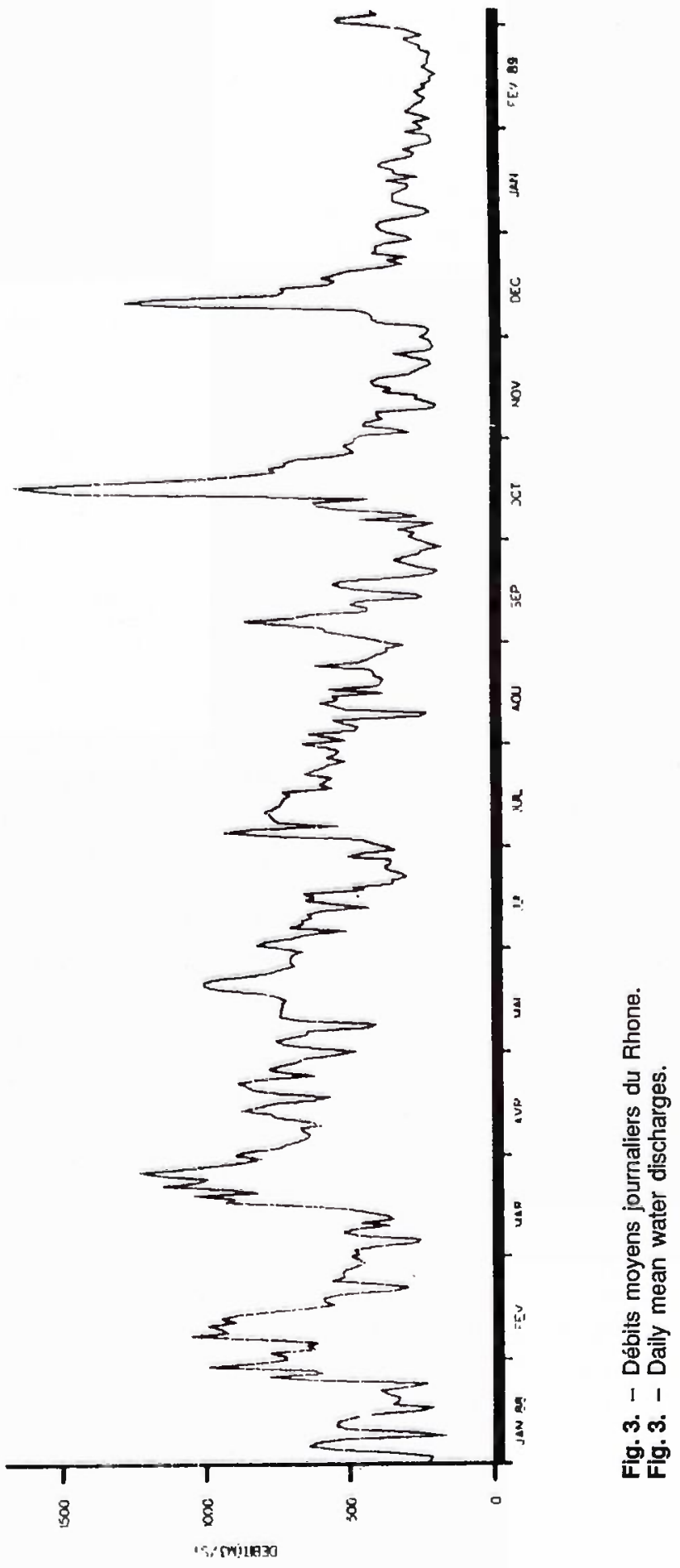


Tableau 3. - Analyses physico-chimiques: Moyenne du secteur en 1988-1989 (3 stations confondues).

Table 3. - Physico-chemical analyses: Mean annual values on the whole sampling area.

\begin{tabular}{|c|c|c|c|c|}
\hline & avril & guin & actobre & février \\
\hline Débit. & 741 & 630 & 480 & 256 \\
\hline $\mathrm{Ph}$ & 8 & 8.1 & 7.8 & 7,7 \\
\hline MES & 25.6 & 12.3 & 17 & 4.3 \\
\hline Conductivité & 280 & 278 & 296 & 316.6 \\
\hline$O_{2}$ & 10.9 & 9,4 & 9.63 & 11,9 \\
\hline $\mathrm{DBO}=$ & 1.86 & 2,5 & - & 3.1 \\
\hline $\cot$ & 1.80 & 2,7 & - & 2.56 \\
\hline $\mathrm{NH}_{4}{ }^{+}$ & 0,14 & 0,17 & 0,19 & 0,23 \\
\hline $\mathrm{NO}_{2}^{-}$ & 0.08 & 0,18 & 0,08 & 0,10 \\
\hline $\mathrm{NO}_{3}$ & 2,9 & 2,6 & 3.23 & 4,68 \\
\hline $\mathrm{PO}_{4} \cdots$ & 0.14 & 0.04 & 0.06 & 0,40 \\
\hline SiO:? & 1.60 & 1.9 & 2,4 & 1,53 \\
\hline $\mathrm{Cl}$ & 5 & 6.3 & 8 & 10.5 \\
\hline $\mathrm{SO}_{4}$ & 40.6 & 52,6 & 39,6 & 48,3 \\
\hline $\mathrm{HCO}_{3}$ & 153 & 147 & 157 & 148 \\
\hline
\end{tabular}

allant de 7,7 à 8,1 , ce qui est conforme avec la nature essentiellement calcaire du bassin versant.

- Conductivité

Le Rhône dans ce secteur apparaît moyennement minéralisé (classes 5-6, Nisbet \& Verneaux 1970). La conductivité est relativement constante durant l'année, les valeurs les plus élevées $(316,6)$ sont notées en février, période de faible débit $\left(256 \mathrm{~m}^{3} / \mathrm{s}\right)$.

\section{- MES}

Les valeurs de MES, relevées au cours des 4 campagnes de prélèvement témoignent d'une situation normale pour le cours d'eau. Les teneurs les plus fortes $(25,6 \mathrm{mg} / \mathrm{l})$ sont relevées en avril lors d'un débit relativement important $\left(740 \mathrm{~m}^{3} / \mathrm{s}\right)$.
- Matières organiques, DB05 et COT

La DB05 est comprise entre 1,8 et $3,1 \mathrm{mg} / \mathrm{l}$. La valeur la plus élevée est observée en février pendant la période d'étiage.

Les teneurs en carbone organique $(1,8-2,7 \mathrm{mg} / \mathrm{l})$ correspondent aux teneurs naturelles des eaux courantes exemptes de matières humiques $(0,5<$ COT $<3 \mathrm{mg} / \mathrm{l})$.

La situation apparaît acceptable sur l'ensemble du secteur pour ces deux variables.

- Sels minéraux $\mathrm{HCO}^{-}, \mathrm{CL}^{-}$, $\mathrm{SO}_{4}^{-}, \mathrm{SiO}_{2}$

Les teneurs observées pour chacun de ces paramètres sont normales pour un grand cours d'eau et traduisent une forte productivité. Cependant il convient de noter, pour la station A 
Tableau 4. - Résultats des analyses physico-chimiques: Moyenne annuelle des trois stations (A : St Vulbas, B : Loyettes RD, C : Loyettes RG).

Table 4. - Results of physico-chemical analyses: Mean values at each sampling asite (A : St Vulbas, B : Loyettes RD, C : Loyettes RG).

\begin{tabular}{|c|c|c|c|}
\hline & \multicolumn{3}{|c|}{ stations } \\
\hline & A & B & C \\
\hline $\mathrm{Ph}$ & 7.9 & 7,97 & 7.92 \\
\hline MES & 14.5 & 15,7 & 14.2 \\
\hline Conductivité & 294 & 291 & 294 \\
\hline $\mathrm{O}_{2}$ & 10.5 & 10,6 & 10.3 \\
\hline $\mathrm{DBO}_{\mathrm{s}}$ & 3.1 & 2 & 2.4 \\
\hline $\mathrm{COT}$ & 2.55 & 2.42 & 2.3 \\
\hline $\mathrm{NH}_{4}{ }^{-1}$ & 0.19 & 0,17 & 0.18 \\
\hline $\mathrm{NO}_{2}=$ & 0.09 & 0.13 & 0.10 \\
\hline $\mathrm{NO}_{3}^{--}$ & 3,01 & 3,3 & 3.76 \\
\hline $\mathrm{PO}_{4}-$ & 0.16 & 0.16 & 0.16 \\
\hline $5 \mathrm{iO}_{2}$ & 1.97 & 1.8 & 1.85 \\
\hline $\mathrm{Cl}^{-}$ & 7,2 & 7.6 & 7,5 \\
\hline $\mathrm{SO}_{4} \cdots$ & 45 & 46 & 45 \\
\hline $\mathrm{HCO}_{3}$ & $15 i$ & 149 & 154 \\
\hline
\end{tabular}

Tableau 5. - Résultats des analyses physico-chimiques: Valeurs annuelles moyennes des paramètres physico-chimiques (1975-1988-89).

Table 5. - Results of physico-chemical analyses: Comparison of physico-chemical variables (1975-1988-89).

\begin{tabular}{|c|c|c|c|c|c|c|}
\hline & $\begin{array}{c}\text { (Loyette } \\
\text { Max }\end{array}$ & $\begin{array}{r}1975 \\
\text { RD ) } 12 \\
\text { Moy }\end{array}$ & $\begin{array}{l}\text { mesures } \\
\text { Mini }\end{array}$ & $\begin{array}{c}\text { (Loyette } \\
\text { Max }\end{array}$ & $\begin{array}{r}1988 \\
\text { RD) } 4 n \\
\text { Moy }\end{array}$ & $\begin{array}{r}\text { mesures } \\
\text { Mini }\end{array}$ \\
\hline $\mathrm{Ph}$ & 8.4 & 8.05 & 7.5 & 8,1 & 7,97 & 7,9 \\
\hline Conductivité & 330 & 283 & 240 & 315 & 291 & 275 \\
\hline $0=$ & 11,2 & 9,2 & 7,7 & 12,2 & 10,6 & 8.8 \\
\hline $\mathrm{HCO}_{3}^{--}$ & 200 & 151,6 & 98 & 155 & 149 & 145 \\
\hline $\mathrm{NH}_{k_{*}}{ }^{+}$ & 0,37 & 0.27 & $>0.06$ & 0,19 & 0,17 & 0,15 \\
\hline $\mathrm{NO}_{2}{ }^{-}$ & 0,13 & 0.06 & 0,03 & 0.20 & 0.13 & 0,08 \\
\hline $\mathrm{NO}_{3}$ & 4.4 & 2.9 & 1,3 & 4,5 & 3,3 & 2,8 \\
\hline $\mathrm{PO}_{2}, \cdots-$ & 0,48 & 0,30 & 0,12 & 0.40 & 0,16 & 0.03 \\
\hline $\mathrm{SiO}_{2}$ & 2.9 & 1.65 & 1 & 2.3 & 1.80 & 1,3 \\
\hline $\mathrm{Cl}^{-}$ & 7,4 & 4,90 & 3,6 & 11 & 7,6 & 5 \\
\hline $\mathrm{SO}_{4} \cdots$ & 48 & 38,7 & 29 & 55 & 46 & 39 \\
\hline $\mathrm{DBO}_{: 3,}$ & 3.7 & 2,6 & 1,5 & 2,5 & 2 & 1,2 \\
\hline
\end{tabular}


(rive droite) des concentrations en $\mathrm{Cl}^{-}$ plus élevées, en liaison avec l'activité industrielle de la plaine de l'Ain.

- Substances azotées

$\mathrm{NH}_{4}^{+}, \mathrm{NO}_{2}^{-}, \mathrm{NO}_{3}^{-}$

Selon les normes Nisbet-Verneaux (1970) les concentrations moyennes relevées en $\mathrm{NH}_{4}^{+}$et $\mathrm{NO}_{2}^{-}(\mathrm{Cl} 2-3)$ sont le signe d'une pollution insidieuse qui apparaît de manière constante sur le secteur. Si l'on se réfère aux grilles adoptées en juin 1990 par les Agences de l'eau (1), ces concentrations correspondent à un milieu modérément pollué.

\section{- $\mathrm{PO}_{4}{ }^{---}$}

Les teneurs en $\mathrm{PO}_{4}{ }^{---}$sont caractéristiques d'eaux fortement productives et modérément polluées (grilles Agences de l'eau 1990).

\section{Conclusion}

Les résultats des analyses mettent en évidence une situation physico-chimique acceptable à l'exception des teneurs excessives en $\mathrm{NH}_{4}{ }^{+}$et $\mathrm{NO}_{2}{ }^{-}$.

La comparaison des résultats obtenus pour chacune des trois stations du secteur ne fait pas apparaître de différence significative d'un point à l'autre (tableau 4).

Cependant, des études récentes, (Mouvet et al., 1986 et rapport Agence de l'Eau RMC, 1988) ont mis en évidence, dans ce secteur du Rhône, la présence de micropolluants. En particulier, à l'amont de St Vulbas, les effluents d'une usine de

(1) Grilles communiquées par le SRAE Rhône-Alpes. traitement de déchets entraînent une forte contamination en $\mathrm{Cu}$ et $\mathrm{Hg}$ se faisant ressentir jusqu'au niveau de Loyettes et nettement plus importante sur la rive droite du fleuve.

Les concentrations moyennes en cuivre, dans des Bryophytes importés (prélèvements réalisés en 1988) passent de $22 \mathrm{mg} / \mathrm{kg}$ de Bryophytes en amont de la zone industrielle à $186 \mathrm{mg} / \mathrm{kg}$ en aval (Loyette rive droite). De même, des teneurs en mercure et en composés organiques de synthèse (PCBS) passent respectivement de 0,16 à $0,69 \mathrm{mg} / \mathrm{kg}$ et de 512 à $890 \mathrm{mg} / \mathrm{g}$.

\subsection{Comparaison entre 1975 et 1988 (tableau 5)}

En 1975 la moyenne annuelle est établie à partir de mesures mensuelles sur la station de Loyettes R.D. En 1988 ces mesures ont été effectuées trimestriellement sur la même station.

En 1988, la qualité physico-chimique des eaux de ce secteur reste comparable à celle de 1975 (classes Nisbet-Verneaux 1970). On note cependant un enrichissement en sels dissous par rapport à 1975, particulièrement en $\mathrm{Cl}^{-}$et $\mathrm{SO}_{4}^{--}$. Le cycle de l'azote, déjà perturbé en 1975 avec des teneurs en $\mathrm{NH}_{4}{ }^{+}$relativement élevées l'est encore en 1988 avec des concentrations non négligeables en $\mathrm{NO}_{2}{ }^{-}$.

Les teneurs en $\mathrm{PO}_{4}^{--}$sont en 1988 sensiblement inférieures à celles de 1975. 
Globalement la qualité physico-chimique, mesurée par les paramètres classiques, ne présente pas de modifications sensibles. Toutefois, il convient de signaler l'existence d'une pollution par des métaux et des composés organiques de synthèse à St Vulbas, provenant de la zone industrielle de la plaine de l'Ain et entraînant une dégradation notable de ce secteur.

\section{3. - BIOLOGIE}

\subsection{Macroinvertébrés benthiques (sauf oligochètes)}

\subsubsection{Evolution des peuplements entre 1975 et 1988}

L'étude de la faune benthique porte sur la totalité des macro-invertébrés récoltés par les prélèvements in situ et à l'aide des substrats artificiels.

Les résultats obtenus en 1977-78 (CTGREF 1977; Perrin 1978) sur le site de St Vulbas sont considérés comme représentatifs de l'ensemble du secteur et successivement confrontés aux données de 19881989 pour chacune des 3 stations (tabl. 6).

\section{- Richesse taxonomique}

En 1988 la station échauffée (station B) est celle qui présente globalement la plus faible richesse spécifique avec 48 espèces ou taxons répertoriés (tab. 7, fig. 4). Le secteur de référence (station $A$ ) renferme 52 espèces et la station $C, 63$ espèces.
En ce qui concerne la campagne d'avril (tabl. 8), la richesse taxonomique est relativement faible sur l'ensemble des 3 stations, mais ceci est dû à l'absence de prélèvements in situ compte tenu des trop forts débits existants à cette période.

Pour les autres dates de prélèvement, on observe au niveau des deux stations aval d'importantes variations du nombre de taxons. Ainsi, la richesse taxonomique maximale apparaît en juin avec 40 et 43 taxons récoltés aux points $A$ et $C$, elle est encore élevée en septembre et diminue fortement en hiver. Cette évolution est liée au cycle de développement des invertébrés.

En revanche, au niveau du secteur échauffé (station B) la richesse taxonomique reste relativement stable de juin à février soulignant ainsi le caractère particulier de ce secteur qui présente un type de fonctionnement différent, l'élévation de température ayant sans doute une influence sur le cycle biologique des organismes.

Le tableau 7 ainsi que la figure 4 mettent en évidence l'évolution de la richesse taxonomique entre les deux périodes d'étude. On observe une nette diminution du nombre d'espèces pour l'ensemble des 3 stations. Cette diminution est plus importante pour le secteur échauffé (st. B) et c'est sur la rive gauche (st. C) qu'elle est la moins sensible (perte de 13 taxons seulement). A elle seule cette baisse de la richesse taxonomique est déjà le signe d'une certaine dégradation du milieu qui semble affecter d'une manière plus intense la station située di- 


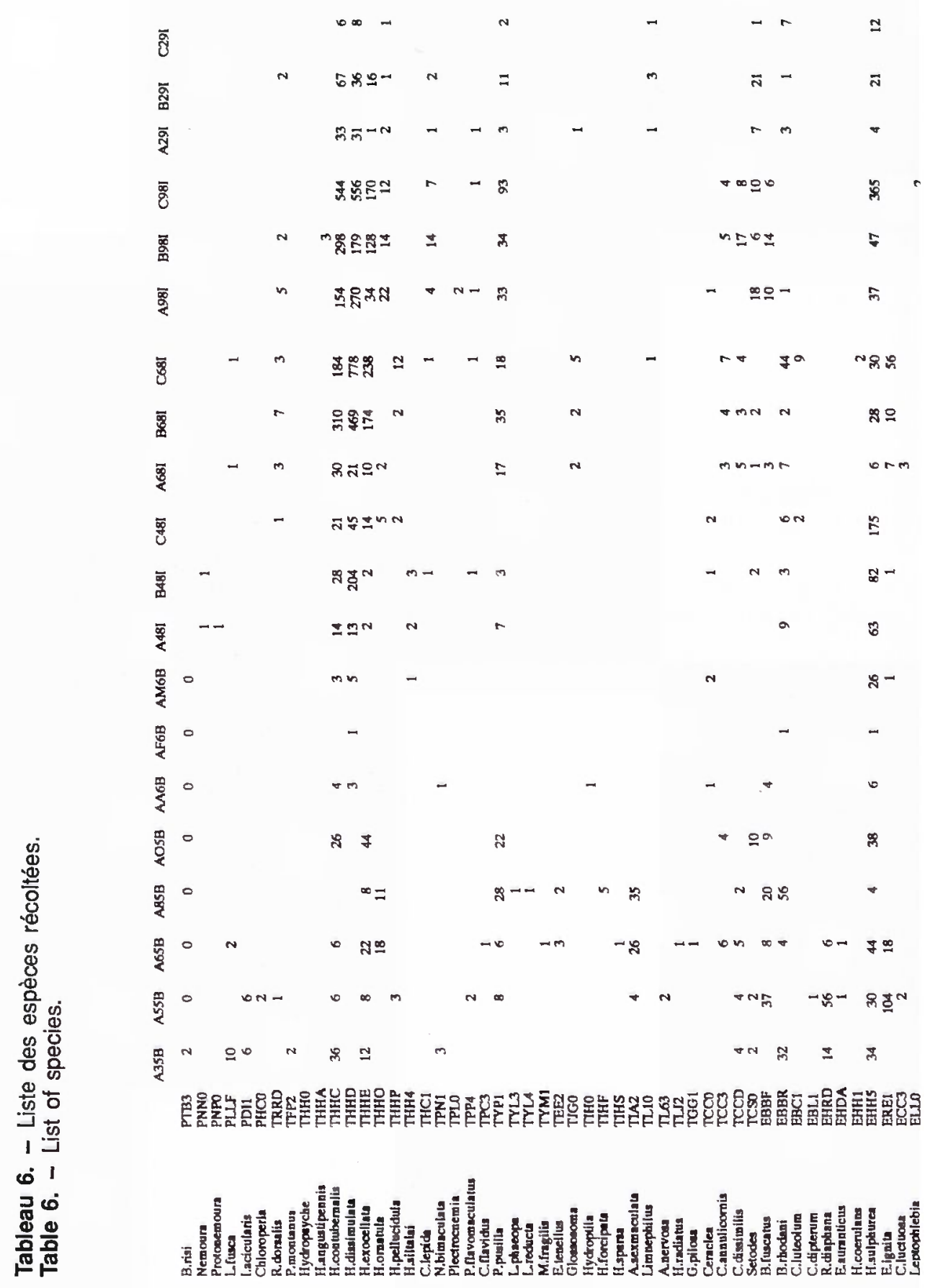


rectement à l'aval du Centre de Production Nucléaire.

La diminution de la richesse taxonomique affecte la grande majorité des ordres étudiés, seules les sangsues et les planaires conservent le même nombre d'unités systématiques (tabl. 7).

Tableau 7. - Richesse taxonomique : évolution entre 1975 et 1988-89.

Table 7. - Taxonomic richness recorded at each study (1975 and 1988-89).

\begin{tabular}{|lccccccccc|}
\hline Station A & Pléco & Tricho & Ephém & Colé & Dipt & Sapla & Moli & Div & Total \\
\hdashline 1975 & 4 & 27 & 10 & 7 & 6 & 6 & 8 & 5 & 74 \\
1988 & 3 & 16 & 5 & 5 & 7 & 6 & 4 & 5 & 52 \\
Taxons en + & 2 & 4 & - & 2 & 2 & 1 & 1 & 2 & 16 \\
Taxons en - & 3 & 15 & 4 & 4 & 1 & 1 & 5 & 2 & 38 \\
& 1 & 11 & 4 & 2 & 1 & - & 4 & - & 22 \\
\hline
\end{tabular}

\begin{tabular}{|lccccccccc|}
\hline Station B & Pléco & Tricho & Ephém & Coléo & Dipt & Sapla & Moll & Div & Total \\
\hdashline 1975 & 4 & 27 & 10 & 7 & 6 & 6 & 8 & 5 & 74 \\
1988 & 4 & 17 & 5 & 5 & 5 & 6 & 4 & 4 & 48 \\
Taxons en + & 1 & 4 & - & 3 & 1 & 1 & - & 1 & 13 \\
Taxons en - & 4 & 14 & 5 & 4 & 2 & 2 & 4 & 2 & 39 \\
& 3 & 10 & 5 & 1 & 1 & - & 4 & 1 & 26 \\
\hline
\end{tabular}

\begin{tabular}{|c|c|c|c|c|c|c|c|c|c|}
\hline Station C & Pléco & Tricho & Ephém & Coléo & Dipt & Sapla & Moll & Div & Total \\
\hline 1975 & 4 & 27 & 10 & 7 & 6 & 6 & 8 & 5 & 74 \\
\hline 1988 & 1 & 15 & 9 & 13 & 7 & 6 & 7 & 5 & 63 \\
\hline Taxons en + & - & 3 & 3 & 7 & 1 & 2 & 4 & 2 & 27 \\
\hline \multirow[t]{2}{*}{ Taxons en - } & 3 & 15 & 4 & 1 & - & 2 & 5 & 2 & 38 \\
\hline & 3 & 12 & 1 & 6 & 1 & - & 1 & - & 11 \\
\hline
\end{tabular}




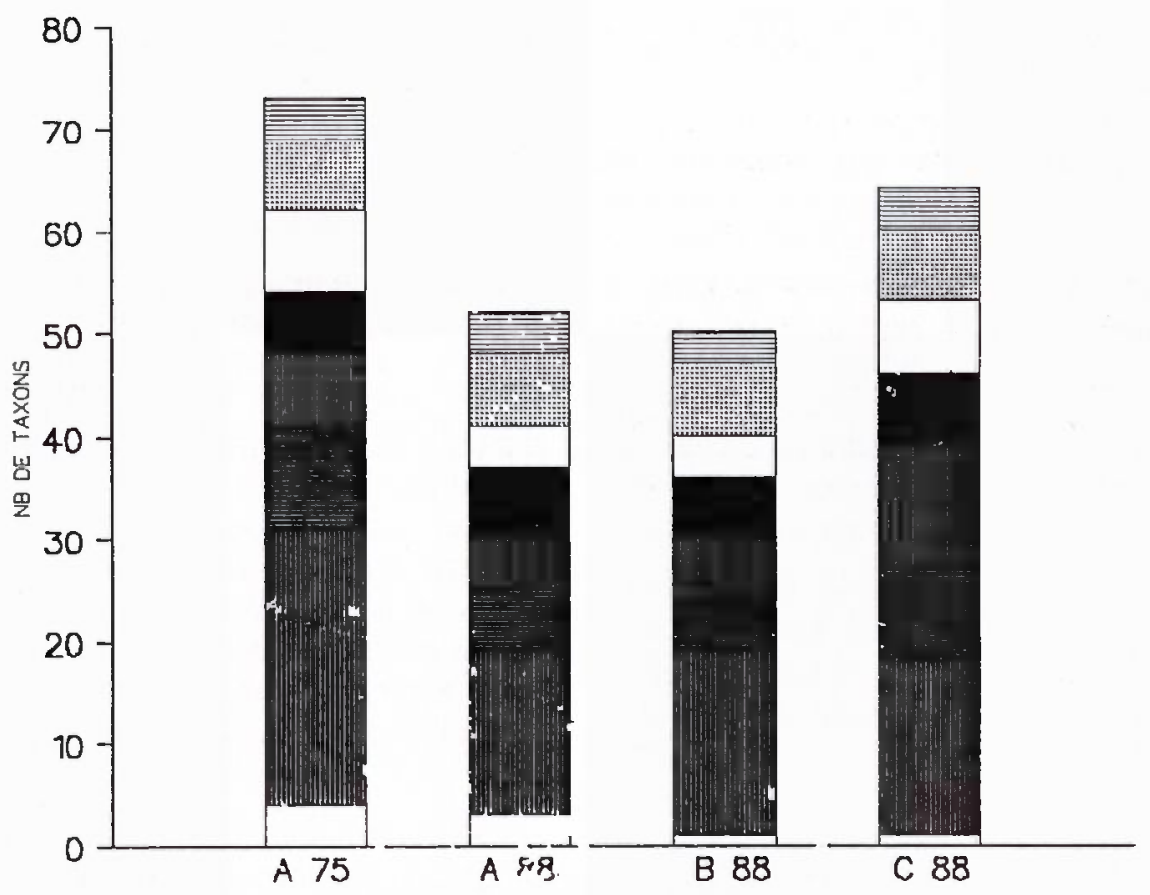

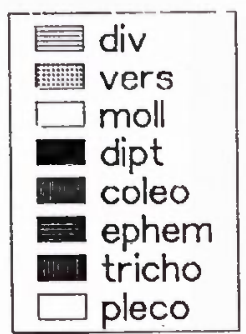

Fig. 4. - Diversité totale.

Fig. 4. - Total number of invertebrate taxa.
En fait, la réduction de la richesse taxonomique ne correspond pas simplement à la perte d'un certain nombre d'espèces mais à une modification plus profonde des biocénoses se traduisant par la disparition et l'apparition de certaines unités systématiques (tabl. 7). Généralement celles-ci ne sont représentées que par un nombre restreint d'individus dont la fréquence est relativement faible.
Ils constituent le cortège des taxons rares et aléatoires. Cependant, dans quelques cas on peut noter la disparition de taxons qui étaient relativement abondants en 1975-1976 (tabl. 6).

Dans l'ordre des plécoptères seul Leuctra fusca est encore récolté sur les stations $A$ et $C$ mais disparaît de la station $\mathrm{B}$; les trois autres espèces disparaissent également partout, elles 
sont remplacées par des Nemouridae (Nemoura, Protonemura) plus polluorésistants.

Les trichoptères qui constituent le groupe le plus diversifié de ce secteur perdent un taxon (Hydroptilidae) qui était particulièrement abondant dans le chenal secondaire à St Vulbas où il colonisait les substrats caillouteux parsemés de débris végétaux en faciès lotique. La disparition plus ou moins totale de ce type d'habitat entraîne l'absence de ces organismes.

Le peuplement d'éphéméroptères encore bien diversifié en 1975-1976 est particulièrement réduit sur les 2 stations de la rive droite avec 6 et 5 espèces, alors que sur la rive gauche (St. C) si l'on constate la perte de cinq unités systématiques, trois nouvelles espèces s'y développent.

Rhithrogena diaphana abondante en 1975-1976 (76 individus) n'est plus récoltée aujourd'hui, cette espèce, sensible à la pollution, paraît être victime de la dégradation de la qualité du milieu. De même, le genre Ecdyonurus a pratiquement disparu.

Le cas de Leptophlebia marginata est particulier: très peu abondante sur la rive droite (4 individus récoltés in situ en 1975) cette espèce présente de fortes densités en rive gauche dans les substrats artificiels : 80 individus en juin 1975 et 60 en avril 1988.

Concernant les coléoptères, bien que la richesse taxonomique reste moyenne, les modifications de ce peuplement sont importantes, particulièrement sur la station $\mathrm{C}$ où la disparition de 6 taxons est compensée par l'apparition de 7 nouvelles unités systématiques d'abondance réduite du fait de la rareté de leurs habitats électifs: faciès lénitique peu représenté, absence de formations végétales en faciès lotique.

Pour les autres groupes d'insectes (odonates, hémiptères, diptères) déjà peu diversifiés en 1975-1976, le nombre de taxons ne change guère. $\mathrm{Ce}$ pendant les odonates semble avoir des difficultés à se maintenir sur le secteur : les 2 espèces recensées en 1975 ne sont plus récoltées actuellement, en liaison avec l'absence d'habitats propices (végétation rivulaire et/ou sédiments grossiers stables en courant modéré) et en liaison également avec le marnage important.

Les mollusques forment également un groupe faunistique dont la richesse spécifique a singulièrement diminué, principalement en rive droite où l'on note la disparition de 5 taxons remplacés seulement par Dreissena polymorpha. Les espèces qui ont déserté ce secteur se distinguent par leur caractère sténotope. Elles sont en effet inféodées à des types d'habitats bien particuliers, représentés dans le faciès lénitique soit par des plantes (cas de Physa acuta et Radix peregra) soit par un substrat vaseux sablo-limoneux pour Unio tumida et Anodonta margaretifera ou encore par ces deux types d'habitats pour Valvata. La disparition progressive de ces habitats et des zones de calme permanentes sont les causes de la raréfaction de ces organismes.

En revanche, sur la rive gauche, la perte de cinq espèces est compen- 
Tableau 8. - Richesse spécifique et abondance des macroinvertébrés récoltés dans le Rhône (site du Bugey), campagne 88-89.

Table 8. - Taxonomic richness and abundance of macroinvertebrates (1988-89).

\begin{tabular}{|c|c|c|c|c|c|c|c|c|c|c|c|c|c|c|}
\hline & & A4BI: & KHUEI & C4BI & AteI & E681 & $\cos \mathrm{I}$ & AรดI & EPQI & C96̈I & Ä29I & E29I & C25I & $\begin{array}{l}\text { Total } \\
\text { taxons }\end{array}$ \\
\hline \multirow[t]{2}{*}{ Flécoptekes } & Fis & 2 & 1 & - & 1 & - & 1 & - & - & - & - & - & -1 & 3 \\
\hline & $\mathrm{Ab}$ & 2 & 1 & - & 1 & - & 1 & - & - & - & - & - & - & \\
\hline \multirow[t]{3}{*}{ Trichoptères } & fis & 5 & 7 & 7 & 10 & 10 & 12 & 11 & 10 & 9) & 10 & 9 & 6 & 17 \\
\hline & $\mathrm{Ab}$ & $3 B$ & 245 & 50 & 110 & 1049 & 1274 & $=144$ & 572 & 1405 & 81 & 157 & 19 & \\
\hline & $\%$ & 0,5 & 2,7 & 2 & 0,4 & 5 & $11, \overline{0}$ & 4,5 & $14, c$ & 12 & 6,4 & 4,1 & 0,7 & \\
\hline \multirow[t]{3}{*}{ Enhtrieroptengs } & FiE & 3 & 3 & 5 & $b$ & 4 & 7 & 4 & 3 & 3 & 3 & 3 & 2 & 10 \\
\hline & $A b$ & 74 & 86 & 244 & 141 & 42 & 243 & E5 & 62 & 37 & $B$ & 23 & 19 & \\
\hline & $\%$ & 1 & 1 & 5,5 & 0,5 & 0,2 & 2,3 & 0,4 & 1,6 & 3,2 & 0,6 & 0,6 & 0.7 & \\
\hline \multirow[t]{2}{*}{ Loleopteres } & $\bar{\hbar} \subseteq$ & 3 & 1 & 1) & 4 & 3 & 6 & 4 & 1 & $t$ & 2 & 4 & 3 & 15 \\
\hline & at & 4 & 1 & 2 & $b$ & $E$ & b) & $\dot{b}$ & 1 & ?) & 5 & 6 & 3 & \\
\hline \multirow[t]{2}{*}{ biotères } & fis & 1 & 1 & 1. & 4 & 3 & 6 & 4 & 3 & 3 & 1 & 2 & - & B \\
\hline & Ab & 1 & 3 & 1 & 22 & 5 & 17 & $b$ & 3 & b) & 2 & 2 & - & \\
\hline \multirow[t]{2}{*}{ [iilrononidae } & $\mathrm{Ab}$ & 452 & 127 & 도. & 1307 & 1076 & 2240 & 116 & 211 & 260 & 29 & 60 & 77 & \\
\hline & $\%$ & 5.8 & 1,5 & 14,8 & 4,2 & 5 & 21,3 & 0,9 & 5.4 & 2,2 & 2.3 & 1,5 & 2,5 & \\
\hline \multirow[t]{2}{*}{ Autres incectes } & Fis & & & & 2 & $i$ & 2 & & & & & $i$ & & 3 \\
\hline & Ab & & & & $b$ & 2 & 2 & & & & & 1 & & \\
\hline \multirow[t]{3}{*}{ Total Insectes } & his & 15 & 16 & 16 & 26 & 22 & 35 & 24 & 18 & 22 & 17 & 20 & 12 & 57 \\
\hline & 触 & 571 & 463 & 587 & 1601 & 2181 & 383 & 725 & 849 & 2005 & 125 & 251 & 118 & \\
\hline & $\%$ & 7.3 & 5,4 & 22,3 & 5,1 & 10,2 & 35,2 & 5,8 & 21,6 & 17,41 & 9,3 & 5,2 & 4,3 & \\
\hline ASEILUS & & & 1 & & 1 & & & 1 & & $1)$ & & 1 & & \\
\hline \multirow[t]{2}{*}{ Ganmarys } & $A b$ & 6715 & 7850 & 2425 & 28260 & $13 \overline{\mathrm{s}} \mathrm{0}$ & $45 j$ & 10503 & 2865 & 5304 & 875 & 3502 & 2263 & \\
\hline & $y$ & $87: 2$ & $5+2$ & 55 & 92,2 & 89 & 59,4 & 90,7 & 73,6 & 79,1 & 70 & 72,1 & 85 & \\
\hline \multirow[t]{3}{*}{ Mollusques } & his & 1 & 1 & 2 & 3 & 3 & 4 & 4 & 3 & 5 & - & 2 & 3 & $\overline{8}$ \\
\hline & Aिb & 1 & 2 & 6 & 56 & 14 & 41 & 5 & 7 & 44 & - & 2 & 5 & \\
\hline & 3 & 0,01 & 0,02 & 0,1 & 0,2 & 0,1 & 0,4 & 0,4 & 0,1 & 0,4 & - & 0,05 & 0,1 & \\
\hline \multirow[t]{2}{*}{ oî igothetes } & A & 415 & 86 & 934 & 697 & 137 & 475 & 210 & 20 & 253 & 205 & 11 & 268 & \\
\hline & $\%$ & 5.3 & 1 & 22,3 & 2,2 & 0,3 & 4,4 & 1.7 & 0,5 & 2,5 & 16,4 & 0,3 & 10 & \\
\hline Sangeues & his & i & 1 & 2 & $\dot{b}$ & 2 & 2 & 4 & 5 & b) & 4 & 4 & 2 & 7 \\
\hline \multirow[t]{2}{*}{ Planaires } & Át & 2 & 2 & 5 & $2 \pi$ & $\underline{B}$ & 7 & 116 & 151 & 36 & 44 & 38 & 8 & \\
\hline & 7 & 0.02 & 0,02 & 0,1 & 0,1 & $0,0 \overline{3}$ & 0,1 & 0,9 & 8 & 0,3 & 3,5 & 1 & 0,3 & \\
\hline Total & fis & 19 & 21 & 22 & 40 & 29 & 45 & 55 & 26 & 37 & 23 & 27 & 19 & 75 \\
\hline Eotnonal & Ât & 7704 & 8236 & 4407 & उos. & $2117 b$ & 10747 & 12012 & 3892 & 11745 & 1247 & 3985 & $2 b 62$ & \\
\hline
\end{tabular}


sée par l'installation de quatre nouveaux taxons, dont deux sont électifs des zones de courant et des substrats durs et stables qui dominent sur cette rive abrupte, $(D$. polymorpha et dans une moindre mesure Bithynia tentaculata).

Le peuplement de sangsues et de planaires ne subit guère de modifications entre les deux périodes d'étude. Deux espèces $H$. stagnalis et $P$. geometra semblent en voie d'extinction sur le secteur alors qu'il est intéressant de noter le développement d'une nouvelle espèce : Dendrocoelum lacteum dont la présence est le signe d'un enrichissement du milieu en matières organiques. Par ailleurs, Dugesia tigrina abondante au niveau de St Vulbas en 1975 présente globalement de plus fortes densités sur le secteur réchauffé (St. 2) avec 133 individus recensés, phénomène en relation sans doute avec l'élévation de température.

- Abondance

Le tableau 8 montre la part importante prise par les gammares.

Sur les 3 stations et à chaque saison ces crustacés sont dominants, leur pourcentage varie de $55 \%$ en avril sur la station $C$ jusqu'à plus de $93 \%$ sur le secteur réchauffé à la même période.

les abondances relatives maximales sont atteintes en juin pour les stations $A$ et $B$, en septembre pour la station C; en septembre le secteur B présente la densité minimale de gammares (2 $\left.865 \mathrm{ind} / \mathrm{m}^{2}\right)$, ce qui est la conséquence des fortes températures estivales entraînant une disparition partielle de ces organismes sur cette station (Roger \& Faessel 1989). L'évolution de la densité des gammares sur les 2 autres stations est en accord avec les observations de nombreux auteurs. Elle est liée au cycle de développement de ces crustacés (Hynes 1955, Roux 1970, Iversen \& Jesson 1977, Dessaix 1980 et Roger \& Faessel 1989) : pas de reproduction en hiver donc effectifs faibles, reprise de l'activité sexuelle dès la fin de l'hiver mise en évidence par une augmentation des effectifs à partir du mois d'avril.

Les autres macro-invertébrés benthiques sont essentiellement représentés par 3 ordres d'insectes (trichoptères, éphéméroptères et Chironomidae) et par les oligochètes, leurs proportions présentent d'importantes variations suivant les saisons et les points d'études.

Au niveau du secteur amont (St $A$ ), le pourcentage d'insectes est faible (tableau 6, fig. 5). Ces organismes sont sensiblement plus abondants en février $(10,1 \%)$. Parallèlement, la proportion des oligochètes atteint $16,4 \%$, alors qu'à cette saison, les gammares ne représentent plus que $70 \%$ de l'abondance totale.

En rive gauche (st C), les insectes, durant le cycle d'étude présentent d'importantes variations de leur abondance relative, le minimum $(4,6 \%)$ étant atteint en février. Au printemps (avril, juin) ce pourcentage devient relativement important $(22,4 \%$ et $35,7 \%$ ). II est dû essentiellement à la prédominance des Chironomidae 
Table 9. - Liste des taxons rares aléatoires.

Table 9. - List of rare taxa.

\begin{tabular}{|c|c|c|}
\hline Station A & Station B & Station $\mathrm{C}$ \\
\hline Nemoura sp. & Nemoura $s p$. & Leuctra sp. \\
\hline Protonemura sp. * & P. flavomaculatus & orechtochilus villosus \\
\hline Leuctra sp. & Dolichopodidae* & Haliplus sp. \\
\hline Limnephilus sp. & Pisidium Sp. & Bidessus sp. ${ }^{*}$ \\
\hline Riolus sp. & Erpobdella sp. & Agabus sp.* \\
\hline Tipulidae & Glossiphonia sp. & Hydraena sp. * \\
\hline Sialis lutaria* & & Riolus sp* \\
\hline Dreissena polymorpha & & Corixa sp. \\
\hline \multirow[t]{6}{*}{ Piscicola geometra ${ }^{*}$} & & Velia sp.* \\
\hline & & Limnomidae \\
\hline & & Psychodidae* \\
\hline & & Gyraulus sp. * \\
\hline & & Sphaerium corneum* \\
\hline & & Erpobdella sp. \\
\hline
\end{tabular}

* taxons présents une seule fois sur le secteur du Bugey et à un exemplaire.

alors qu'en septembre ce sont au contraire les trichoptères qui dominent.

Sur la station échauffée (st. B) on peut noter une variation plus faible du pourcentage des insectes en fonction des saisons. En effet, les insectes sont bien représentés en juin et deviennent importants $(22 \%)$ en septembre, essentiellement grâce à la forte densité des trichoptères et à la faible densité des gammares.

Si l'on considère les taxons rares et aléatoires (cf. Richesse taxonomique) c'est en rive gauche (station $\mathrm{C}$ ) qu'ils se révèlent les plus nombreux (15 taxons recensés).

Ils sont surtout représentés par des coléoptères ( 7 genres ou espèces) à caractère plutôt lénitophile en liaison sans doute avec la présence d'une petite noue située en amont de la zone de prélèvement. En revanche, au niveau du secteur réchauffé, ces taxons «rares» sont les moins nombreux, six seulement ayant été répertoriés.

Par ailleurs, pour le total des quatre campagnes de prélèvements on a pu identifier un certain nombre de taxons propres à chacune des stations (tabl. 10). La rive gauche se distingue encore par un nombre relativement élevé (17) de taxons propres, particulièrement au mois de juin alors que le secteur réchauffé n'en comporte que 3 .

La comparaison avec les années 1975-76 montre pour la station A une faible modification des densités (fig. 5). Les éphéméroptères et les diptères diminuent sensiblement alors que les trichoptères et les oligochètes augmentent; cette situation s'accentue sur la station soumise à l'échauf- 
Tableau 10. - Liste des taxons propres ) chaque station.

Table 10. - List of characteristic taxa of each sampling sites.

\begin{tabular}{|c|c|c|}
\hline Station A & Station B & Station C \\
\hline $\begin{array}{l}\text { Protonemura sp. } \\
\text { Plectrocnemia sp. } \\
\text { C. luctuosa } \\
\text { S. lutaria } \\
\text { P. geometra }\end{array}$ & $\begin{array}{l}\text { H. angustipennis } \\
\text { Dolichopodidae } \\
\text { P. peregra }\end{array}$ & $\begin{array}{l}\text { C. luteolum } \\
\text { H. coerulans } \\
\text { Leptophlebia sp. } \\
\text { Habrophiebia sp. } \\
\text { Bidessus sp. } \\
\text { Agabus sp. } \\
\text { Hydraena sp. } \\
\text { Dryops sp } \\
\text { Limnius sp. } \\
\text { o. tuberculatus } \\
\text { Riolus sp } \\
\text { Stenelmis sp. } \\
\text { Velia sp. } \\
\text { P.sychodidae } \\
\text { Gyraulus sp. } \\
\text { B. tentaculata } \\
\text { S. corneum }\end{array}$ \\
\hline
\end{tabular}

fement (St. B) sauf en ce qui concerne les oligochètes qui perdent de leur importance. En revanche sur la rive gauche (St. C) on constate une forte augmentation de l'abondance totale, de l'ensemble des groupes considérés et plus particulièrement des diptères Chironomidae et des oligochètes. Sur tout le secteur, il convient de signaler une très forte augmentation de l'abondance des gammares.

Globalement, entre $1975-1976$ et la période actuelle, l'accroissement des effectifs moyens par station est généralement dû à la prolifération des trichoptères Hydropsychidae, des Chironomidae et des Gammaridae. Le peuplement d'oligochètes, constitué dans le cas présent par des orga- nismes de taille supérieure à $600 \mu \mathrm{m}$ (protocole de prélèvement IQBG) et considéré globalement sur chacune des stations présente également des abondances plus élevées en 1988. Ceci n'est pas valable lorsque l'on étudie ces organismes dans les substrats grossiers (cf. chap. oligochètes). La prolifération de ces organismes détritivores et saprobiontes est révélatrice d'une augmentation de la charge organique entre les deux périodes d'étude, particulièrement nette sur la rive gauche où l'on peut noter l'arrivée d'effluents de nature organique (abattoirs...). Cependant cette charge organique n'est pas détectée par les analyses physico-chimiques classiques. 


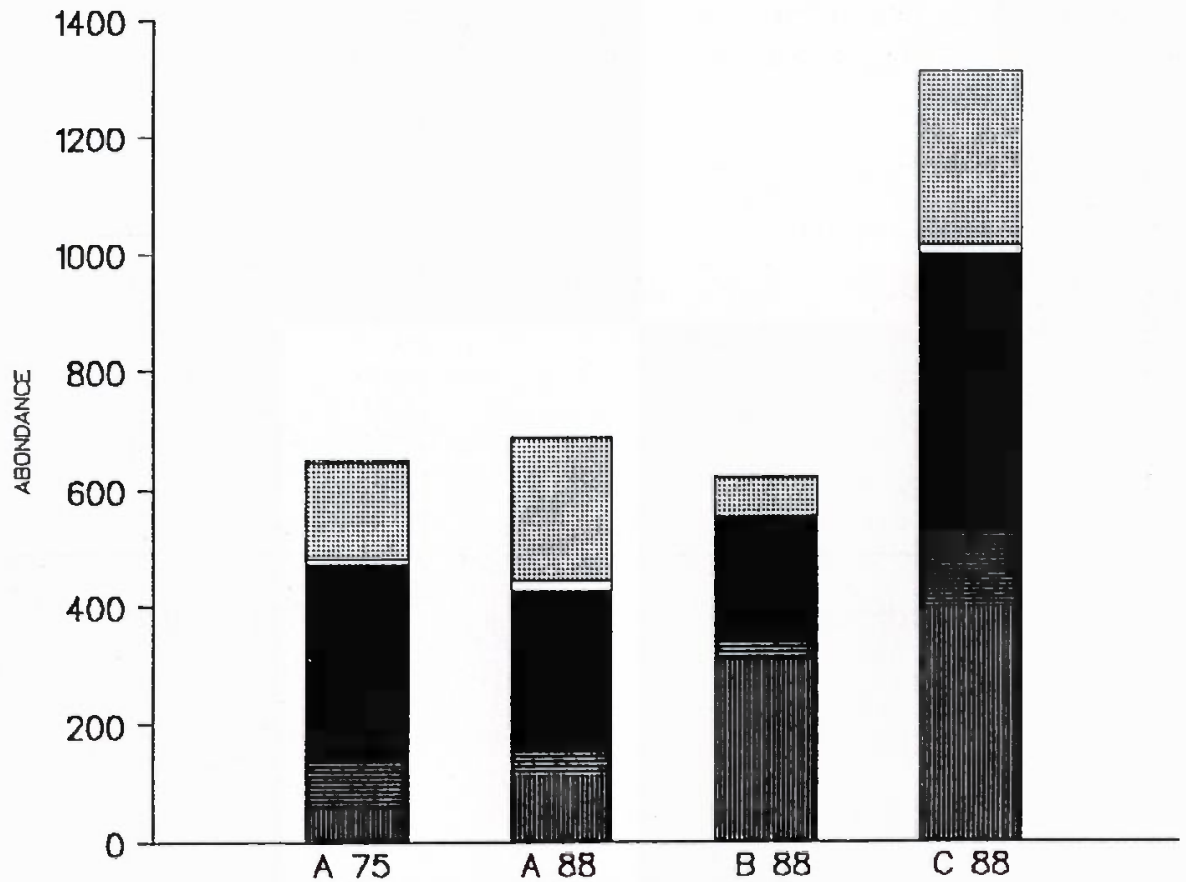

Fig. 5. - Abandance moyenne (sauf Gammaridae).

Fig. 5. - Mean abundances of invertebrates (except Gammaridae).

\begin{tabular}{|l|}
\hline div \\
$\square$ moll \\
$\square$ dipt \\
coleo \\
ephem \\
tricho \\
$\square$ pleco
\end{tabular}

\subsubsection{Effet du réchauffement sur le secteur}

\section{1:2.1. Etude faunistique}

De nombreux auteurs (Arthur et al. 1982, Dusoge \& Wisniewsky 1976, Langford 1972, Roger \& Faessel 1989, Talmage \& Coutant 1980 , Vannote \& Sweeny $1980 .$. ) ont montré que l'augmentation de température engendre des modifications de la dy- namique des peuplements. L'étude détaillée de l'évolution de certaines espèces d'insectes, bien représentées sur les 3 stations, devrait permettre la mise en évidence d'un impact éventuel du réchauffement de l'eau.

\section{Les trichoptères}

Leur abondance est la plus faible au niveau de la station de référence (fig. 6), bien que le nombre d'espèces 

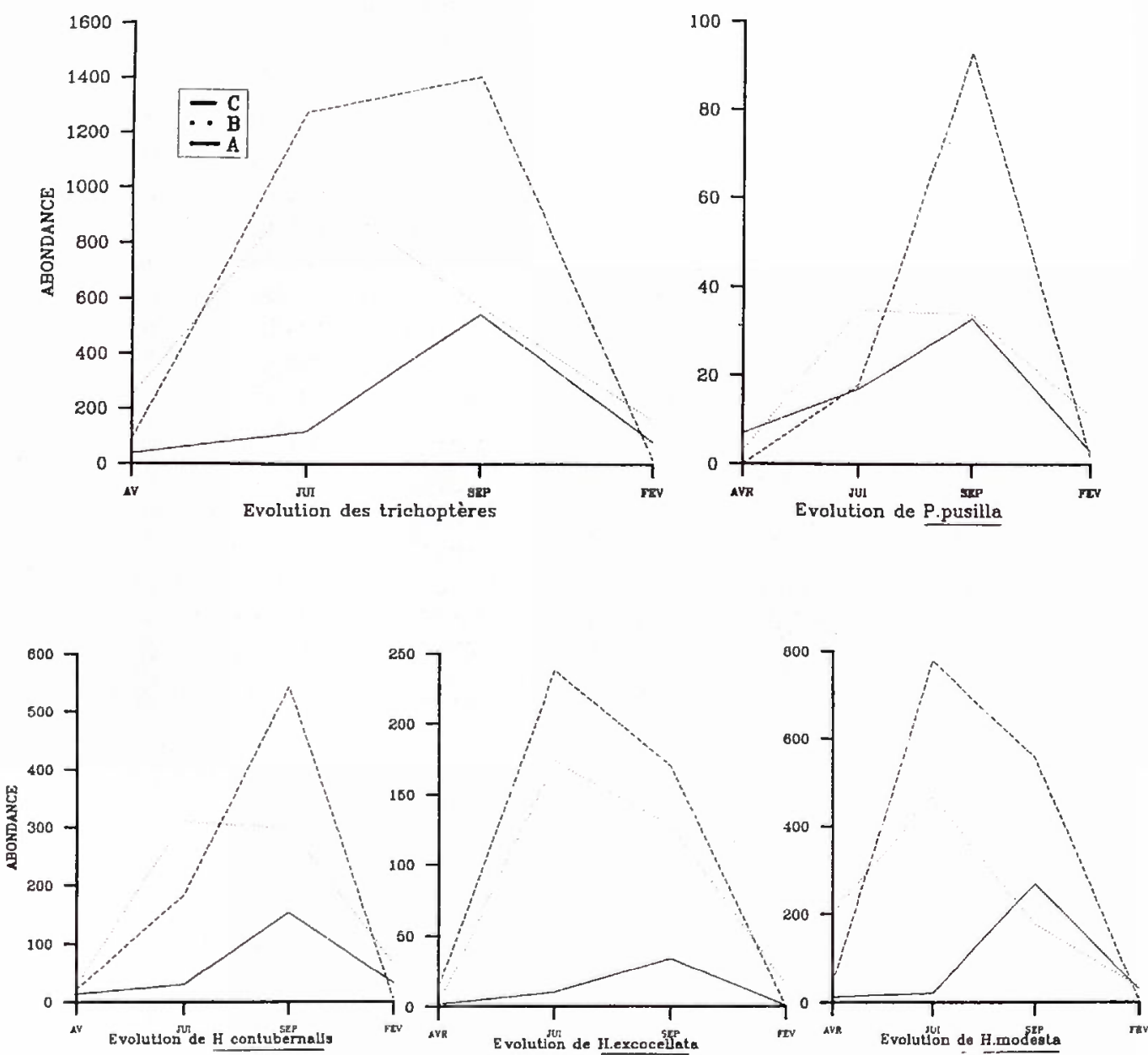

Fig. 6. - Trichoptères : évolution saisonnière des principales espèces.

Fig. 6. - Seasonal variation of the abundance of several Tripchoptera species.

récoltées sur ce site ne diffère guère de ce qui est observé sur les deux autres points d'étude. Cette faible abondance est peut-être à relier aux rejets de la zone industrielle de la plaine de l'Ain, la rive droite étant le siège d'une pollution métallique notable.
Sur l'ensemble du secteur, les trichoptères sont surtout représentés par les Hydropsychidae (8 espèces), par Psychomyiia pusilla et par des Leptoceridae.

L'évolution temporelle de trois espèces d'Hydropsyche, récoltées en 
toutes saisons sur les 3 stations est analysée (fig. 6). Ce sont elles qui déterminent les fortes densités observées pour cet ordre: il s'agit de Hydropsyche contubernalis, Hydropsyche exocellata, Hydropsyche modesta (= dissimulata) espèces bivoltines sur le Rhône (Tachet \& Bournaud 1981).

Le genre Hydropsyche est bien représenté dans le cours supérieur du Rhône où il se développe durant toute l'année avec généralement des effectif́s plus élevés en automne (Bournaud et al. 1982, Gaschignard-Fossati \& Berly 1987).

Ceci peut être observé (fig. 6) pour $H$. conturbenalis sur les stations $A$ et $C$ alors que sur le secteur échauffé (St. B.) cette espèce atteint sa densité maximale dès le mois de juin. II semble donc que $H$. contubernalis soit une espèce sensible à une élévation de la température qui influe sur son cycle de développement. Un tel phénomène a été constaté par ailleurs (Fey 1977) pour Hydropsyche pellucidula qui présente un décalage d'environ deux mois pour les individus ayant atteint le $5^{e}$ stade larvaire entre un secteur réchauffé $\left(\Delta t=6,5^{\circ} \mathrm{C}\right)$ et un secteur témoin.

Le trichoptère $H$. modesta, espèce la plus abondante sur les 3 stations prospectées a ses plus fortes densités en septembre sur le secteur amont et en juin sur les deux stations aval de la centrale. Dans la zone la plus réchauffée (station B) l'abondance de cette espèce diminue brutalement en septembre (fig. 6), soit par suite d'une période d'émergence écourtée, soit à cause de la mortalité des larves, en relation avec les fortes températures relevées en été.

Quant à $H$. exocellata, peu abondante, elle présente les mêmes profils sur les 2 stations aval (B et $C$ ). Sa très faible abondance sur la station amont pourrait être liée à la dégradation de ce secteur, cette espèce étant peut-être plus sensible à la présence de polluants métalliques (Cu-Mg) que les autres espèces d'Hydropsyche.

Conformément aux observations de Gaschignard-Fossati \& Berly (1987) sur le Rhône c'est en été que $P$. pusilla présente les plus fortes densités qui apparaissent dès le mois de juin au niveau de la station échauffée, peut-être en relation avec l'élévation de température (cf. $H$. contubernalis).

Enfin il convient également de souligner, au niveau du secteur échauffé, une plus forte densité des trichoptères durant la période hivernale, sans doute liée à un développement continu de ces organismes, les phénomènes de diapauses pouvant être écourtés par suite du réchauffement.

\section{Les éphéméroptères}

Sur les 10 espèces d'éphéméroptères répertoriées, 3 ou 4 seulement méritent d'être prises en considération en raison de leur fréquence et de leur abondance élevées.

Ainsi Heptagenia sulphurea, Potamanthus luteus et Baetis rhodani peuvent actuellement être considérées comme les éphéméroptères communes et caractéristiques de ce secteur du Rhône. Si l'on se reporte aux données antérieures (CEMA- 

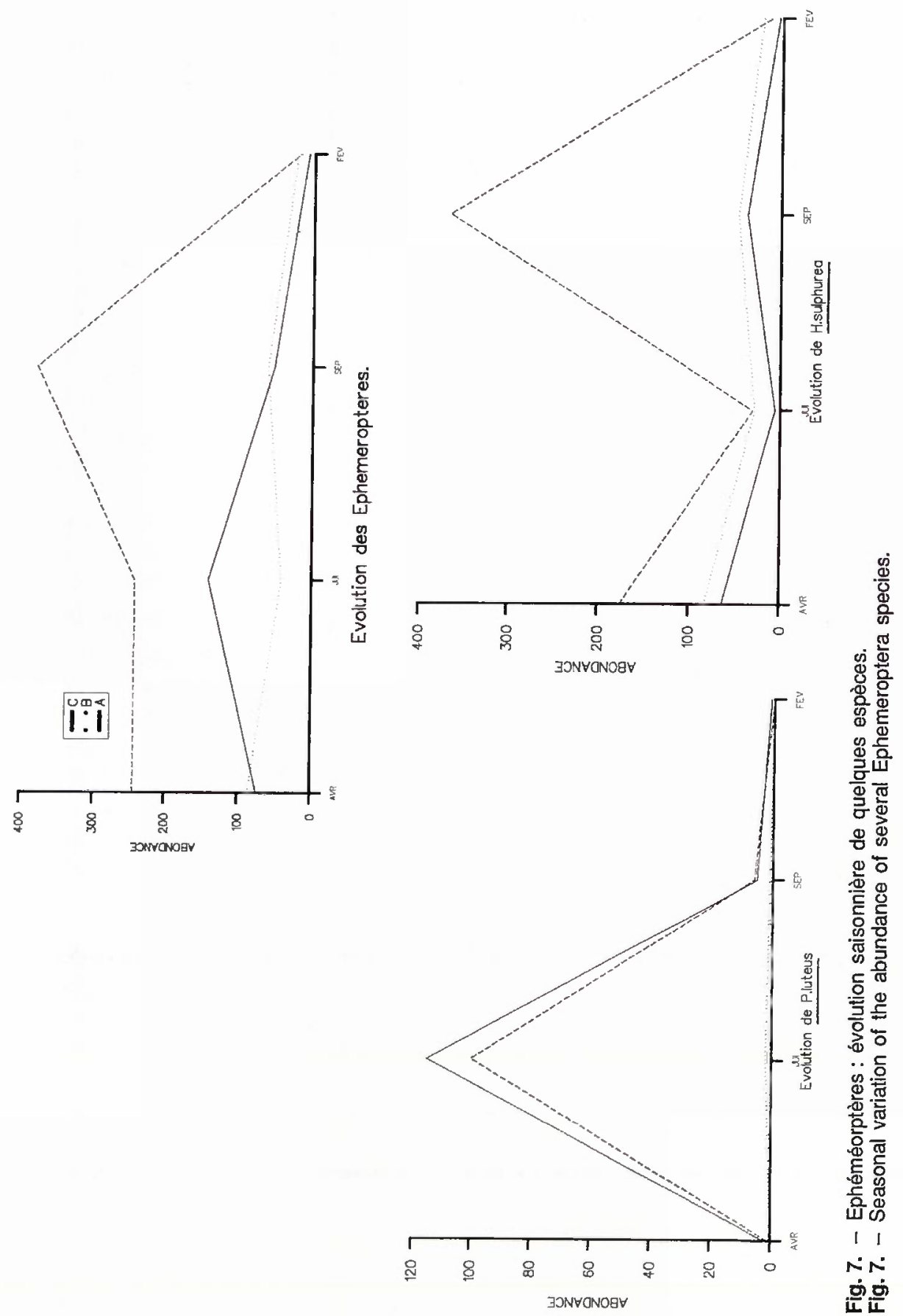
GREF, 1977), à ces 3 espèces s'ajoutaient alors Baetis fuscatus et le genre Rhithrogena bien représenté au printemps.

Le cas de $H$. sulphurea est particulièrement intéressant à étudier (fig. 7, tabl. 6). Cette espèce, bien représentée sur les 3 stations, présente une abondance plus élevée en rive gauche (st. C). L'évolution saisonnière des abondances reflète assez bien le cycle de développement de cette espèce univoltine dans le Rhône (El Kallab-Wakim 1978). Au printemps on observe une forte densité larvaire suivie de faibles effectifs en été, en relation avec la période d'envol de ces insectes, qui s'étale de mai à octobre (El Kallab-Wakim op. cité). Les abondances notables observées en septembre correspondent aux larves provenant des premières pontes de l'été. Le cycle de développement de $H$. sulphurea ne semble pas affecté par l'élévation de température au niveau de la station $B$, tout au plus peut-on remarquer, sur ce secteur, que la reproduction serait plus continue que sur les 2 autres stations caractérisées par des effectifs plus réduits en hiver (tabl. 6), en relation avec le phénomène de quiescence hivernale qui concerne le stade embryonnaire et retarde l'éclosion des larves. Degrange (1959) a en effet mis en évidence un arrêt du développement embryonnaire de cette espèce pour une température inférieure à $10^{\circ} \mathrm{C}$ (arrêt qui ne concerne pas tous les œufs d'une même ponte). Dès que la température augmente, les éclosions reprennent après un temps de latence de quelques jours.
Le cas de $P$. luteus est très différent : bien que présente sur toutes les stations, seul le mois de juin montre une abondance notable de cette espèce sur les sites $A$ et $C$ (fig. 7). En revanche, sur le secteur réchauffé 1 à 2 individus seulement sont recensés au cours de l'année; l'élévation de température relevée sur ce point semble donc inhiber le développement de $P$. luteus. Ces observations concordent avec celles de Obrdjik et al. (1979) sur la rivière Oslava (Tché coslovaquie) recevant un effluent de centrale thermique, les abondances et la production de $P$. luteus s'avérant négligeables dans le secteur réchauffé.

\section{Les diptères}

Ils sont largement dominés par la famille des Chironomidae dont l'abondance, sur les 3 stations est maximale en juin et minimale en février. Au printemps et en été c'est au niveau de la station $B$ que l'on observe les plus faibles densités de Chironomidae, peut être en relation avec des émergences précoces dues au réchauffement du secteur. Parmi les autres diptères récoltés sur cette partie du Rhône, les Simuliidae et les Empididae bien que peu abondants ont une fréquence supérieure à $50 \%$, les seconds (formes prédatrices) se nourrissant souvent aux dépens des premiers (Vaillant 1962).

\subsubsection{Analyse multivariée}

II paraît intéressant de compléter cette étude faunistique par une analyse synthétique de la structure de peuplements. Cette analyse fait appel 


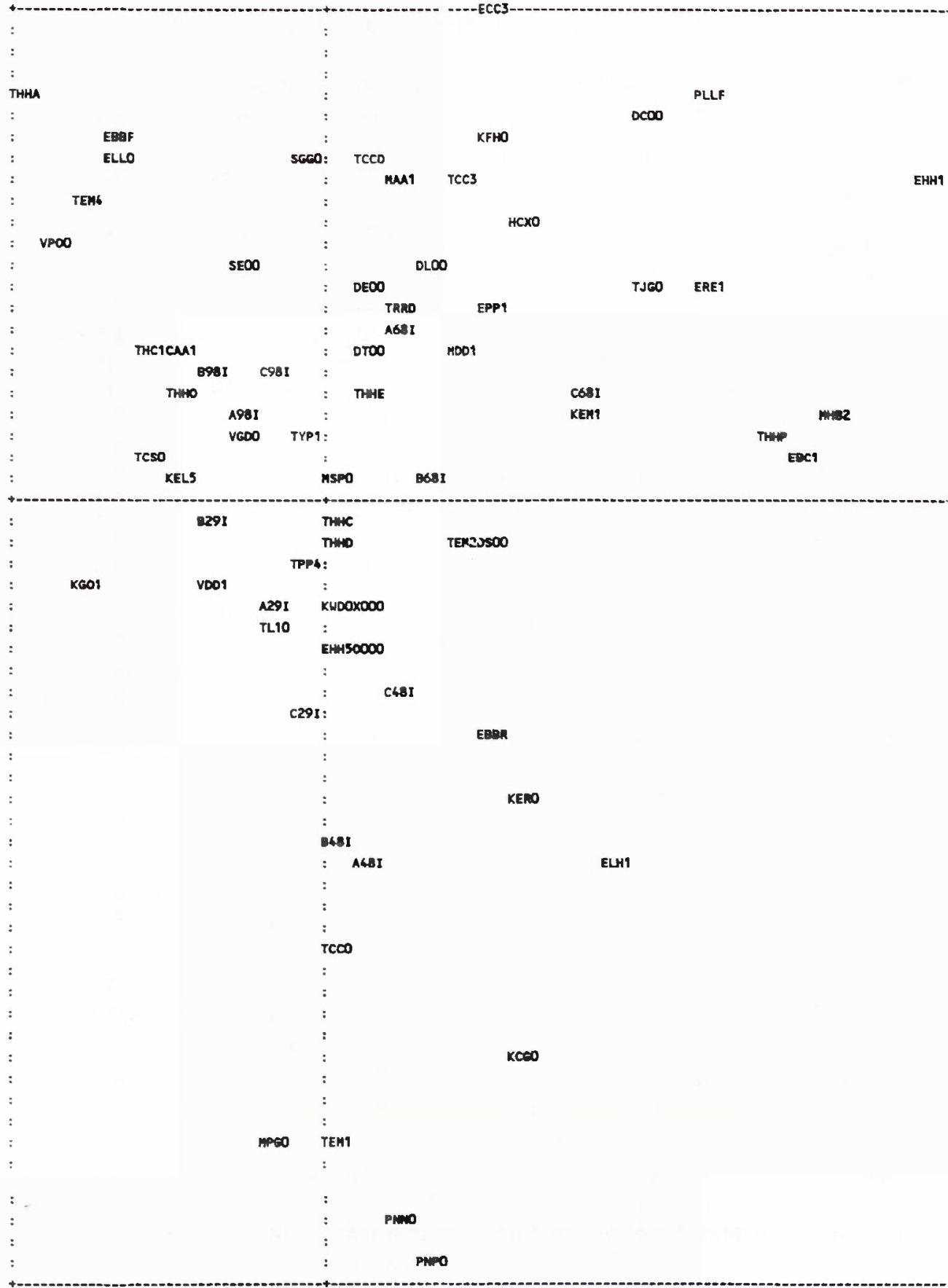

MOManE DE POINTS SUPERPOSES : 23

IHS2(ECC3) SPP1(ECC3) DOOO(TAMA) TEM5(THMA) KELO(ELLO) KESO(ELLO) HSS1(ELLO) KHEO(EHH1) KIHO(EHH1) KEO4(EHHI)

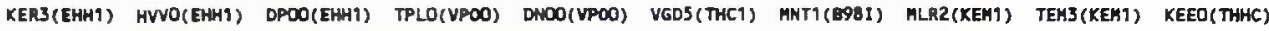
KEFO(AZ9I) COEO(KLDO) THHS(PHAO)

Fig. 8. - Distribution des stations-dates et des espèces dans le plan F1-F2 de l'analyse factorielle. Fig. 8. - Distribution of the station-dates and of the species in the F1-F2 plane of the Factorial Correspondance Analysis (FCA). 
au logiciel ADDAD d'analyse factorielle des correspondances (A.F.C.).

Celle-ci a été effectuée sur un tableau de contingence comprenant 12 lignes (dates, stations) et 81 colonnes (espèces d'insectes et variables thermique).

Les abondances des espèces ont été transformées en log10 afin de minimiser la variabilité inhérente à l'échantillonnage. De plus cette transformation permet d'atténuer le poids des espèces très abondantes et de mieux prendre en compte les espèces moins abondantes. Les taxons représentés par un seul individu ou récoltés une seule fois sur les 12 prélèvements sont mis en éléments supplémentaires.

La variable thermique, également mise en donnée supplémentaire, est représentée par la somme des degrés-jours calculée sur les 3 mois précédents les dates de prélèvement sur chacune des stations. Pour la station C, la somme des degrés-jours a été estimée en tenant compte d'une augmentation de $0,6^{\circ} \mathrm{C}$ par rapport à la station A (cf. chap. 2.2). Les valeur ainsi obtenues sont réparties en 5 classes.

La répartition des stations sur le plan factoriel défini par les axes F1F2, représentant plus de $45 \%$ de l'inertie, montre une évolution saisonnière des biocénoses comparable pour les 3 stations (fig. 8). La prédominance du facteur saisonnier a déjà été soulignée par Perrin (1976) sur le Rhône et par Doledec (1987) sur le cours inférieur de l'Ardèche.
La représentation des coordonnées moyennes des stations sur le facteur F1 en fonction des saisons (fig. 9) met en évidence une opposition entre le mois de juin et le mois de septembre, et dans une moindre mesure entre juin et février.

La courbe des coordonnées moyennes des saisons sur le facteur $\mathrm{F} 1$ en fonction des stations (fig. 10) isole la station $\mathrm{C}$ des deux autres, plus particulièrement au mois de juin en rapport avec la présence sur cette station de quelques taxons $(H$. pellucidula, Centroptilum luteolum et $B$. tentaculata) qui présentent les plus fortes contributions.

Les mêmes analyses effectuées sur le facteur F2 montrent une opposition entre la "saison froide" (avrilfévrier) et la "saison chaude" (juin-septembre (fig. 11). En revanche ce facteur ne permet pas de différencier les stations : elles ont une position comparable par rapport à cet axe (fig. 12).

Une classification ascendante hiérarchique effectuée sur les quatre premiers facteurs de l'AFC $68 \%$ de l'inertie) à partir des coordonnées factorielles des espèces permet de séparer 8 groupes faunistiques répartis en fonction de leurs abondances à certaines périodes de l'année (fig. 13).

Le groupe 1, proche de TEM1 qui est bien représentée sur F2, comprend 2 taxons $H$. siltalai et Protonomura $s p$. qui contribuent fortement à cet axes. Ils peuvent être qualifiés de "taxons froids", $H$. siltalai étant classée dans les espèces d'Hy- 


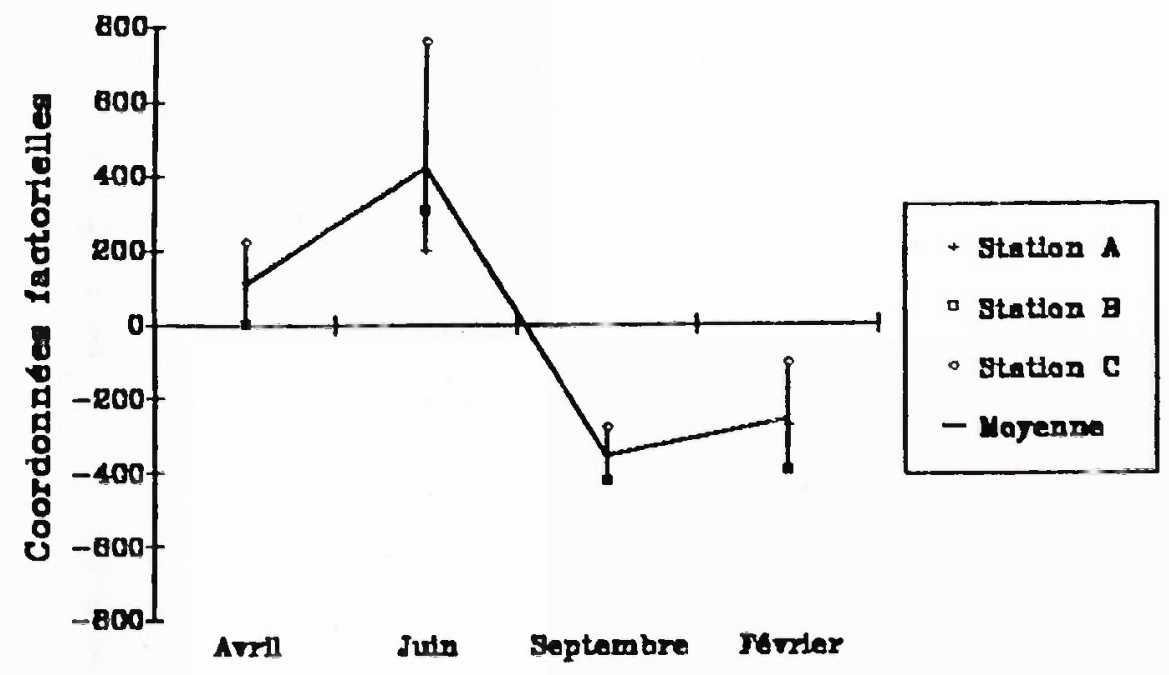

Fig. 9. - Moyenne des coordonnées des stations par saison sur l'axe Fi.

Fig. 9. - Mean values of sampling sites coordinates with the FCA first factor at each sampling season.

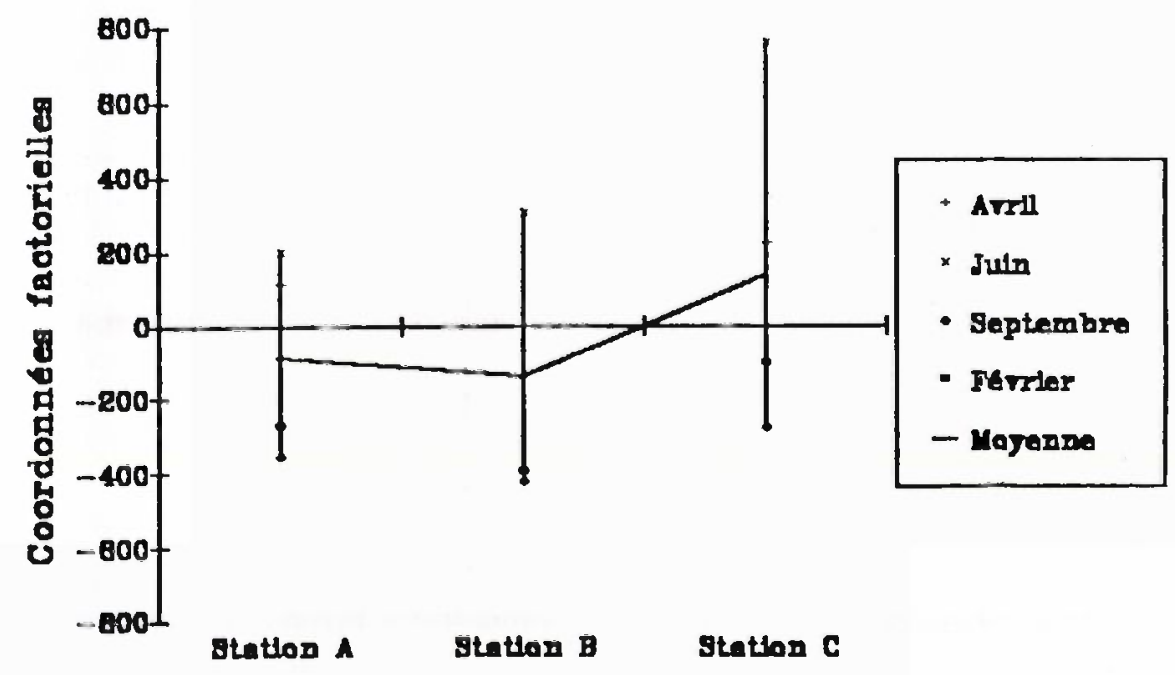

Fig. 10. - Moyenne des coordonnées des saisons par station sur l'axe F1.

Fig. 10. - Mean values of sampling seasons coordinates with the FCA first factor at each sampling station. 


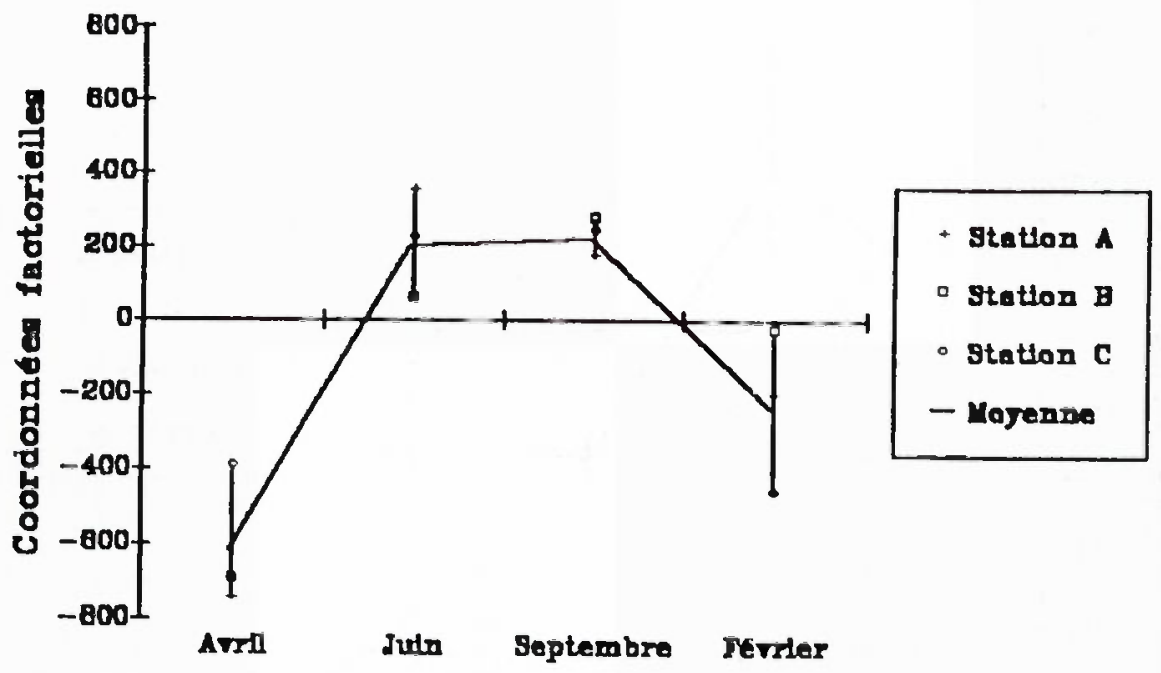

Fig. 11. - Moyenne des coordonnées des stations par saison sur l'axe F2.

Fig. 11. - Mean values of sampling sites coordinates with the FCA second factor at each sampling season.

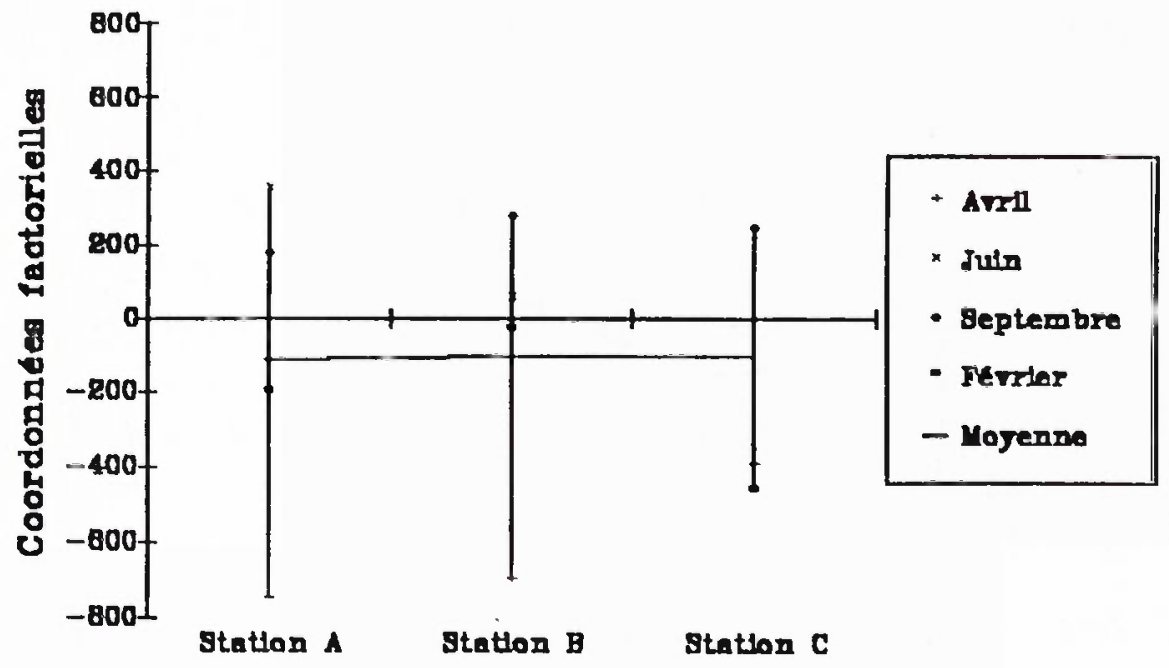

Fig. 12. - Moyenne des coordonnées des saisons par station sur l'axe F2.

Fig. 12. - \%Mean values of sampling seasons coordinates with the FCA second factor at each sampling station. 


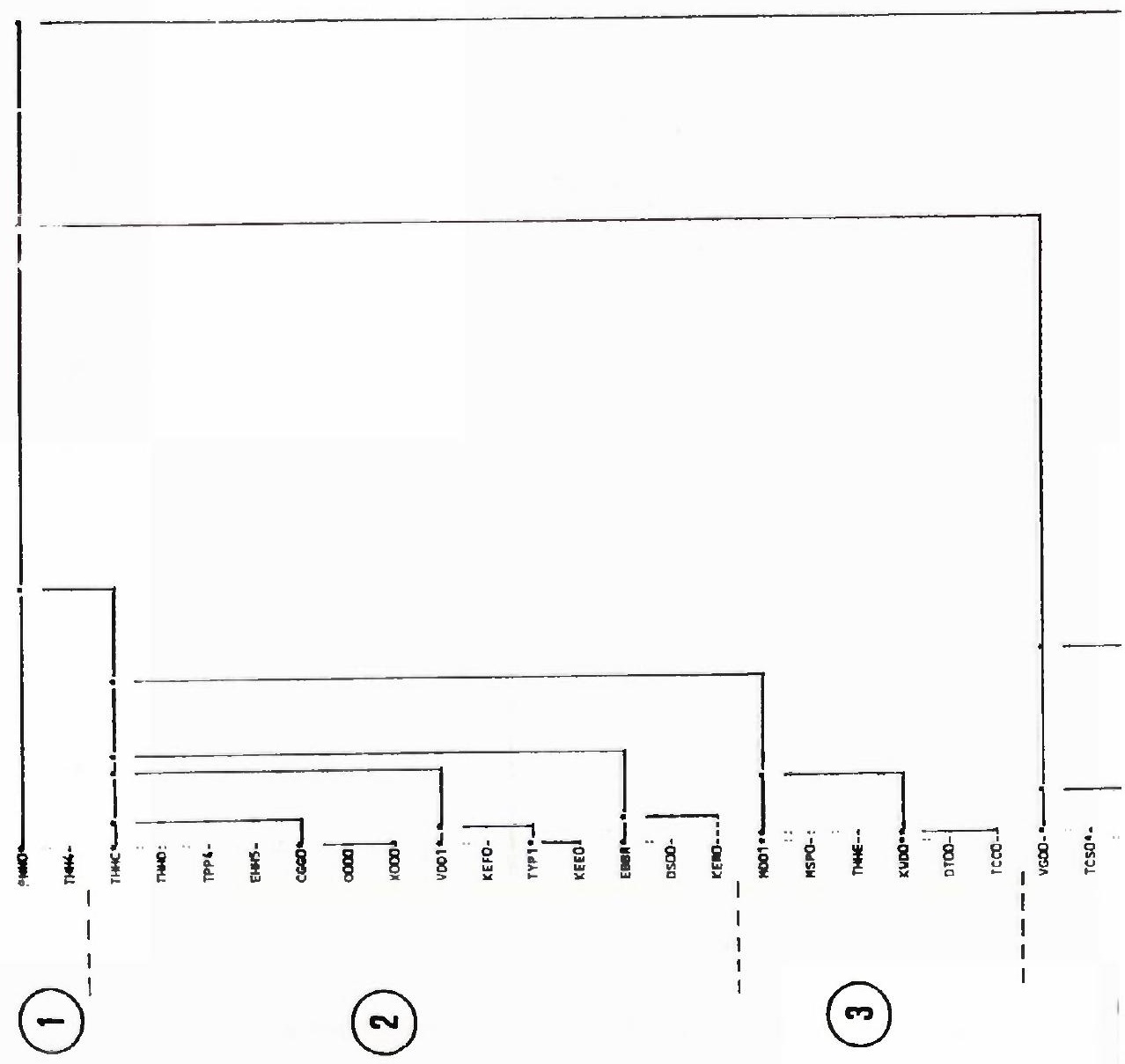




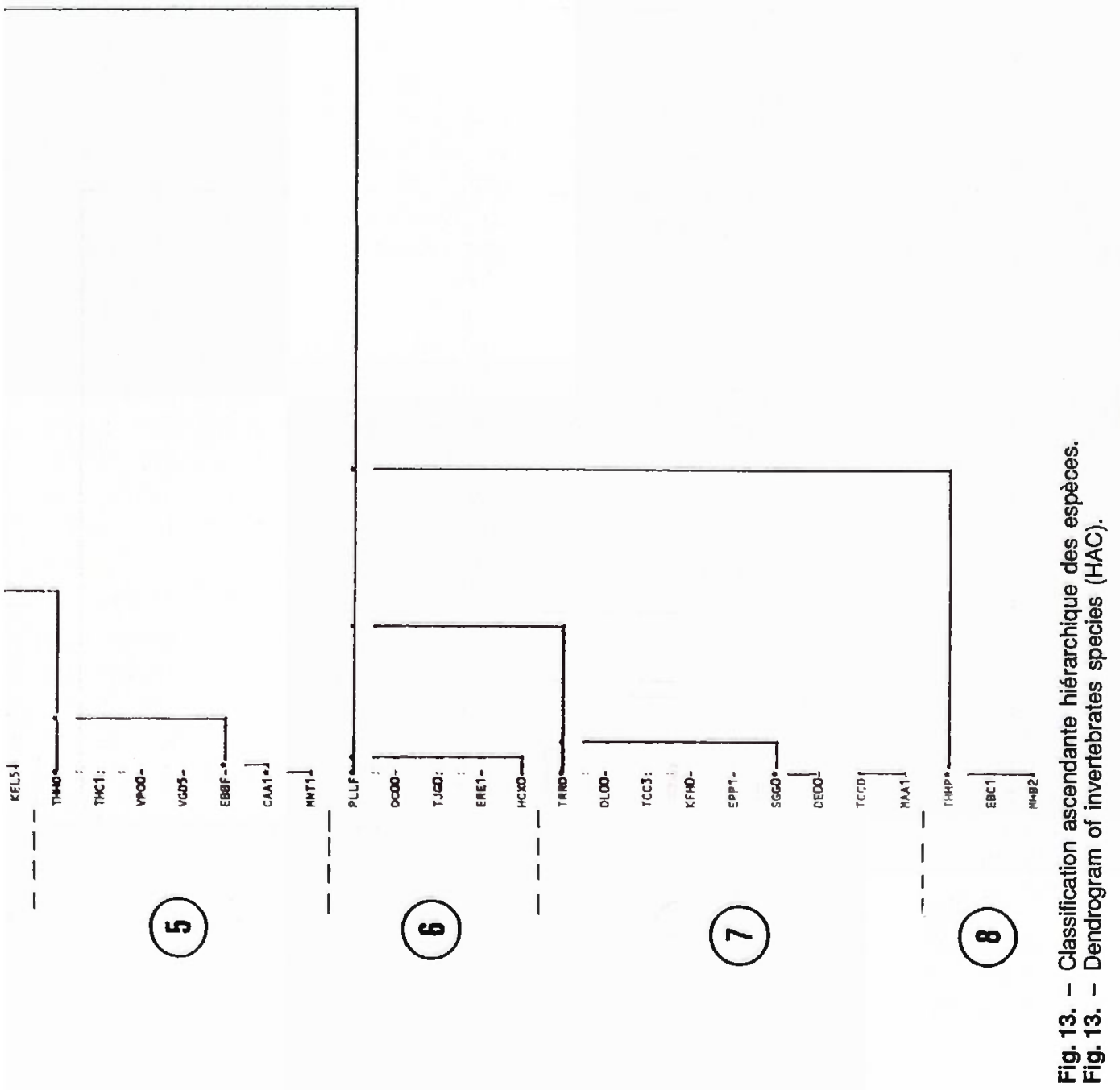


dropsychidae à preferendum froid par Tachet et Bournaud (1981).

Les groupements 2 et 3 les plus proches de l'origine des axes (fig. 8 et 13) comprennent des taxons bien représentés toute l'année sur les 3 stations ou des taxons peu abondants et ne présentant pas de variations saisonnières nettement marquées.

les groupes 4 et 5 sont composés de taxons plus abondants en septembre et février :

- le groupe 5 comporte principalement des espèces automnales ( $\mathrm{Du}$ gesia tigrina, Cheumatopsyche lepida, $B$. fuscatus, $H$. ornatula), les 3 premières étant plus abondantes à la station $B$ où la température est plus élevée; leur abondance diminue notablement en hiver.

- les taxons du groupe 4 sont en général peu abondants, seuls les genres Setodes et Dugesia sont bien représentés; la densité des taxons de ce groupe varie peu entre septembre et février.

Les groupes 6, 8 rassemblent les espèces estivales (juin) le groupe 7 constituant la transition entre les biocénoses estivales (groupes 6,8 ) et les biocénoses automnales (groupe 5).

le groupe 8 est particulier, il comprend 3 espèces inféodées à la station C.

Cette analyse a permis de mettre en évidence une évolution saisonnière des biocénoses comparable pour les 3 stations et une particularité de la station $C$. En revanche, ce type d'analyse globale n'a pas permis de mettre en évidence un effet du réchauffement de l'eau sur les biocénoses du secteur. Cette perturbation n'apparaît donc pas déterminante par rapport à d'autres types "d'agression» qui ont déjà profondément modifié les biocénoses en place, celles-ci n'étant plus composées que d'organismes euryèces.

\subsubsection{Conclusions}

Les quatre campagnes de prélèvements effectuées sur le secteur du Bugey en 1988 ont permis de recenser 75 espèces ou taxons (tabl. 6 et tabl. 7) dont la plupart sont communs sur le cours supérieur du Rhône français (Rapport CTGREF 1977, Perrin \& Roux 1978, Cellot 1982, Gaschignard-Fossati \& Berly 1987, Bournaud et al. 1987 a et b).

Entre 1975 et 1988 on peut noter, sur ce secteur, la disparition de 29 taxons qui, à l'exception des Hydroptilidae et du genre Rhithrogena étaient très peu abondants. En revanche, il y a apparition de 31 taxons également très peu représentés.

En 1988 les insectes forment plus de $77 \%$ de la richesse taxonomique observée sur cette partie du fleuve avec 57 taxons. Les trichoptères, éphéméroptères et coléoptères sont les ordres les plus diversifiés (2) (tabl. 7 et tabl. 8), alors que les plécoptères constituent ici un groupe relique avec seulement 3 genres répertoriés.

La confrontation de la diversité des principaux ordres d'insectes récoltés

(2) Ne tenant pas compte des Chironomidae non déterminés. 
Tableau 11. - Comparaison de la richesse spécifique de 4 ordres d'insectes sur des secteurs de cours d'eau appartenant à la même zone écologique (niveau typologique =7; (3) (4) (2) nombre de stations considérées.

Table 11. - Specific richness of four aquatic insect orers recorded in the typological level B7 (Epipotamon) of six rivers; (3), (4), (2): number of investigated stations.

\begin{tabular}{|lcccccccc|}
\hline Cours d'eau : & $\begin{array}{c}\text { Aube } 1977 \\
\text { (Aube) } \\
\text { (3) }\end{array}$ & $\begin{array}{c}\text { (Aube) } \\
\text { (3) }\end{array}$ & $\begin{array}{c}\text { Loue } \\
\text { (Jura) } \\
\text { (4) }\end{array}$ & $\begin{array}{c}\text { Ognon } \\
\text { (Hte Saóne) } \\
\text { (2) }\end{array}$ & $\begin{array}{c}\text { Dordogne } \\
\text { (Dordogne) } \\
\text { (2) }\end{array}$ & $\begin{array}{c}\text { Rhóne } \\
\text { (19)-1976 }\end{array}$ & $\begin{array}{c}\text { Rhōne } \\
1988-1989\end{array}$ \\
\hline Plécoptères & 4 & 6 & 11 & 2 & 3 & 5 & 3 \\
Trichoptères & 16 & 34 & 32 & 15 & 31 & 22 & 17 \\
Ephéméroptères & 18 & 25 & 20 & 16 & 17 & 10 & 10 \\
Coléoptères & 34 & 51 & $?$ & 3 & 7 & 8 & 15 \\
\hline
\end{tabular}

sur cette partie du Rhône avec des données recueillies sur des stations de même niveau typologique (B7) (Verneaux, 1973) et appartenant à d'autres cours d'eau (tabl. 11), met en évidence la pauvreté de la faune dans ce fleuve. La même remarque peut être faite relativement aux mollusques dont la richesse spécifique s'écarte notablement de la répartition théorique décrite par Mouthon, (1981).

Plusieurs causes peuvent simultanément être à l'origine de la faible richesse taxonomique de la faune benthique sur ce secteur du Rhône.

D'une part la qualité physico-chimique du milieu reste médiocre (cf. chap. 2).

D'autre part il convient de signaler l'existence de nombreux barrages (Verbois, Génissiat, Seyssel, SaultBrenaz) destinés à la production d'énergie électrique et à la maîtrise du débit du fleuve. On sait que de tels aménagements sont susceptibles d'entraîner des modifications importantes des caractéristiques morphody- namiques du milieu (William \& Wolman 1984), de la qualité physico-chimique des eaux (Munn \& Brusnen 1987), du régime thermique et des biocénoses aquatiques (Ward \& Stanford 1979, Scullion et al. 1982-1983, Hauers \& Stanford 1982).

De plus, dans le cas particulier du Rhône, la gestion de ces barrages se traduit par des variations brutales et importantes du débit (fig. 3) qui présentent généralement une influence néfaste sur l'ensemble de la faune benthique (Rapport CTGREF 1977).

Ces variations de débit, souvent assimilables à des crues, se traduisent par une instabilité du substrat qui, conjuguée avec les phénomènes d'entraînement des invertébrés, constituent une des causes importantes de la diminution de la faune benthique, tant en ce qui concerne la richesse spécifique que l'abondance (Gaschignard 1984b, 1986, Gaschignard-Fossati \& Berly 1987, Munn \& Brusven 1987). 
Tableau 12. - Effets du réchauffement sur certains taxons.

Table 12. - Impact of warmed effluents on the distribution of several species or taxa.

\begin{tabular}{|c|c|}
\hline Taxons & Conséquences du réchauffement \\
\hline $\begin{array}{l}\text { Trichoptères } \\
\text { H. contubernalis } \\
\text { P. pusilla }\end{array}$ & $\begin{array}{l}\text { Plus forte abondance en hiver (diapause écourtée). } \\
\text { Abondance maximale plus "tôt (avril). }\end{array}$ \\
\hline $\begin{array}{l}\text { Hodesta } \\
\text { c. lepida }\end{array}$ & $\begin{array}{l}\text { Forte diminution de } 1 \text { 'abondance en septembre. } \\
\text { Plus forte abondance. }\end{array}$ \\
\hline H. sulphurea & $\begin{array}{l}\text { Moins de variations saisonnières d'abondance } \\
\text { (pas d'arrêt du développement embryonnaire). }\end{array}$ \\
\hline P. luteus & Disparition (inhibition). \\
\hline Chironomidae & Diminution d'abondance en avril (et juin) \\
\hline Gammarus & Diminution d'abondance en septembre. \\
\hline $\begin{array}{l}\text { D. tigrina } \\
\text { Pisidium }\end{array}$ & Plus forte abondance. \\
\hline $\begin{array}{l}\text { Ancylus fluviatilis) } \\
\text { (Glossiphonia) }\end{array}$ & Abondance plus faible. \\
\hline
\end{tabular}

Enfin, l'instabilité du substrat nuit au développement des végétaux supérieurs et donc aux macro-invertébrés par suite d'une réduction d'habitats potentiels. De la même façon, le marnage important inhibe l'installation d'une flore rivulaire et entraîne, par la mise en assec de certaines zones, la mort de nombreux organismes, (rapport CTGREF 1977).

Toutes ces observations tendent à montrer un appauvrissement de la faune du secteur qui est essentiellement composée d'organismes urésistants" et ubiquistes et de quelques taxons rares ou reliques. Une situation semblable a récemment été décrite dans les secteurs aménagés du bas-Rhône (Fruget, 1989). II paraît difficile dans ce cas de noter un effet important du fonctionnement du Centre de Production Nucléaire sur les biocénoses, celui-ci pouvant être masqué par les perturbations déjà existantes.

Cependant la plus faible richesse taxonomique observée sur la station $B$ et la dynamique de répartition de quelques espèces (tabl. 12) semblent directement imputables à l'élévation de température.

\subsection{Etude des peuplements d'oligochètes}

\subsubsection{Liste des espèces récoltées}

Au moins vingt-neuf espèces ou taxons ont été recensés à partir des 15 relevés.

L'examen de la liste faunistique (tabl. 13), où les taxons sont notés en présence $\left({ }^{*}\right)-$ absence $(-)$, per- 
Tableau 13. - Liste des espèces d'oligochètes récoltées sur le site du Bugey avec leur code d'identification; les données sont exprimées en présence (") ou absence (-).

Table 13. - List of Oligochaeta species with their identification codes; data are converted into presence $\left(^{*}\right)$ and absence $(-)$.

\begin{tabular}{|c|c|c|c|c|}
\hline ESPECES ET TAXONS & CODES & $\mathbf{A}$ & c & B \\
\hline Lumbriculidae immatures & STOO & * & * & $\star$ \\
\hline Chaetogaster diaphanus & CHDI & $\star$ & $\star$ & $\star$ \\
\hline Nais behningi & NABE & * & * & $\star$ \\
\hline Nais bretscheri & NABR & $\star$ & $\star$ & $\star$ \\
\hline Nais elinguis & NAEL & * & 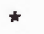 & $\star$ \\
\hline Nais pardalis & NAPA & $\star$ & 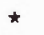 & $\star$ \\
\hline Enchytraeidae g. sp. & ENCH & * & * & $\star$ \\
\hline Cernosvitovielia sp. & CEOO & $\star$ & * & $\star$ \\
\hline Propappus volki. & PPVO & * & * & $\star$ \\
\hline Marionina argentea & MAAR & $\star$ & * & $\star$ \\
\hline Stylodrilus heringianus & STHE & * & \pm & - \\
\hline Tubificidae imm. sans s.c. & TUBC & * & * & - \\
\hline Vejdovskyella intermedia & VEIN & $\star$ & * & - \\
\hline 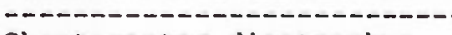 & $---\infty$ & & & \\
\hline Chaetogaster diastrophus & CHDS & - & * & * \\
\hline Nais alpina & NAAL & - & * & * \\
\hline Paranais frici & PAFR & * & _ & * \\
\hline Lumbricidae g. sp. & LUBC & * & - & * \\
\hline Eiseniella tetraedra & EITE & * & - & * \\
\hline Lumbricillus sp. & LCOO & * & - & * \\
\hline Lumbricillus fennicus & LCFE & * & - & * \\
\hline Bythonomus lemani & BYLE & $\star$ & - & - \\
\hline Tubificidae imm, avec s.c. & TUSS & * & - & - \\
\hline Psammoryctides barbatus & PSBA & * & - & - \\
\hline Limnodrilus hoffmeisteri & LIHO & * & - & - \\
\hline Potamothrix moldaviensis & POMO & * & - & - \\
\hline Nais barbata & NABA & * & - & - \\
\hline Phallodrilus sp. & PHOO & - & - & * \\
\hline Nais communis & NACO & - & - & * \\
\hline Marionina riparia & MARI & - & - & * \\
\hline
\end{tabular}

met de mettre en évidence quelques tendances générales:

- parmi les 29 taxons répertoriés, 10 colonisent les trois stations $(A, B$, C), en particulier Propappus volki, Cernosvitoviella sp. et Marionina argentea, qui sont considérés comme des organismes décrivant une activation des échanges hydrodynamiques entre la nappe et le système superfi- ciel (Gaschignard-Fossati 1986, Lafont 1989, Lafont \& Durbec 1990a);

- six taxons ne sont répertoriés que sur la station amont $(A)$ et 3 ne sont rencontrés que sur la station réchauffée (B), où l'on constate la présence du genre Phallodrilus, taxon dont le caractère descripteur est identique à celui des espèces précédemment citées (Lafont \& Durbec 1990b); 
- enfin, 9 taxons, présents sur la station $C$ et/ou sur la station amont (A), désertent le secteur aval réchauffé en rive droite (B).

Ce premier aperçu suggère que, si l'élévation de la température des eaux joue probablement un rôle important dans la distribution des oligochètes, les perturbations hydrauliques engendrées par la gestion des barrages amont présentent également un impact notable.

\subsubsection{Réactualisation des données}

Ce chapitre ne concerne que la station amont $(A)$, où l'on dispose de données récoltées à 10 ans d'intervalle. L'analyse portera sur les espèces les plus fréquentes dans l'ensemble des relevés, soit les espèces ou taxons récoltés au moins trois fois (tabl. 14).

En première analyse, on constate que les effectifs d'oligochètes accusent une baisse sensible entre 1975 et 1987-89. Cette observation s'applique, en toute logique, aux espèces ou taxons les plus communs et les plus abondants (Stylodrilus heringianus et formes immatures du genre Stylodrilus, Propappus volki, Lumbricillus fennicus, Enchytraeidae, Eiseniella tetraedra). Le Lumbricidae E. tetraedra n'a pas été récolté en 1987-89. En revanche, le genre Cernosvitoviella et l'Enchytraeidae Marionina argentea sont recensés pour la première fois en 1987-89. Ces deux taxons sont considérés comme décrivant

Tableau 14. - Données brutes concernant les peuplements d'oligochètes; NSP : nombre d'espèces; EFBR : effectif́s d'oligochètes $/ 0,1 \mathrm{~m}^{2}$; pour les codes d'identification des espèces et taxons, se référer au tableau 13.

Table 14. - Abundance of oligochaetes; NSP : number of species; EFBR : abundance of oligochaetes $/ 0.1 \mathrm{~m}^{2}$; the identification codes refer to table 13.

ST. NSP EFBR STOO STHE ENCH PPVO NAEL CEOO LCFE LCOO NABR NAPA NAAL CHDI NABE MAAR EITE CHDS

\begin{tabular}{rrrrrrrrrrrrrrrrrrrr}
\hline$A 55$ & 07 & 338 & 1 & 1 & 060 & 0 & 019 & 0 & 250 & 0 & 0 & 0 & 0 & 0 & 1 & 0 & 5 & 0 \\
A85 & 08 & 187 & 010 & 086 & 040 & 012 & 0 & 0 & 017 & 0 & 0 & 010 & 0 & 0 & 0 & 0 & 1 & 0 \\
A05 & 06 & 330 & 036 & 025 & 040 & 205 & 0 & 0 & 020 & 0 & 0 & 0 & 0 & 0 & 0 & 0 & 010 & 0 \\
A37 & 10 & 206 & 6 & 3 & 013 & 9 & 034 & 3 & 105 & 024 & 2 & 0 & 0 & 0 & 0 & 0 & 0 & 0 \\
A68 & 09 & 124 & 1 & 0 & 046 & 2 & 1 & 4 & 8 & 058 & 0 & 0 & 0 & 2 & 0 & 0 & 0 & 0 \\
A98 & 07 & 060 & 6 & 4 & 2 & 043 & 0 & 1 & 0 & 1 & 0 & 0 & 0 & 0 & 0 & 2 & 0 & 0 \\
A29 & 07 & 096 & 026 & 1 & 1 & 049 & 2 & 1 & 1 & 3 & 0 & 0 & 0 & 0 & 0 & 012 & 0 & 0 \\
B37 & 10 & 205 & 0 & 0 & 073 & 0 & 045 & 8 & 020 & 8 & 018 & 012 & 2 & 0 & 0 & 6 & 0 & 0 \\
B68 & 12 & 052 & 0 & 0 & 1 & 1 & 1 & 2 & 0 & 0 & 020 & 1 & 7 & 2 & 011 & 0 & 2 & 3 \\
B98 & 02 & 2 & 1 & 0 & 0 & 0 & 0 & 0 & 0 & 0 & 0 & 0 & 0 & 0 & 0 & 0 & 0 & 0 \\
B29 & 09 & 041 & 1 & 0 & 0 & 0 & 023 & 1 & 0 & 0 & 5 & 2 & 2 & 1 & 0 & 0 & 0 & 5 \\
C37 & 10 & 192 & 046 & 7 & 042 & 043 & 031 & 1 & 0 & 0 & 0 & 011 & 1 & 0 & 0 & 6 & 0 & 2 \\
C68 & 06 & 025 & 0 & 0 & 1 & 0 & 0 & 0 & 0 & 0 & 8 & 2 & 7 & 4 & 3 & 0 & 0 & 0 \\
C98 & 04 & 088 & 7 & 010 & 0 & 067 & 0 & 0 & 0 & 0 & 0 & 0 & 0 & 0 & 3 & 0 & 0 & 0 \\
C29 & 04 & 141 & 016 & 5 & 2 & 117 & 0 & 0 & 0 & 0 & 1 & 0 & 0 & 0 & 0 & 0 & 0 & 0
\end{tabular}


l'existence d'échanges actifs entre les eaux superficielles et souterraines (Lafont 1989).

En conclusion, à 10 années d'intervalle, les peuplements d'oligochètes présentent malgré tout une relative stabilité sur le site amont. Les effets des perturbations de nature hydraulique, liées aux aménagements situés en amont de Saint-Vulbas, semblent être les principaux responsables des quelques différences constatées. De plus, la diminution des effectifs pourrait être liée à une dégradation de la qualité physico-chimique des eaux.

A l'appui de ce premier examen, il pouvait s'avérer intéressant d'avoir un aperçu plus synthétique, incluant l'ensemble des données récoltées sur le Rhône, soit les 15 relevés et les 16 espèces ou taxons les plus fréquents (tabl. 14). Les effectifs des espèces ou taxons (transformés en log10) constituent les variables actives d'une ACP normée (logiciel ADDAD).

- Résultats de l'analyse

Le premier facteur ( $32 \%$ de la variance totale) est expliqué par une opposition entre le genre Stylodrilus et $P$. volki et des représentants de la famille des Naididae (Nais brestcheri, $N$. alpina, N. behningi, Chaetogaster diaphanus, $C$. diastrophus, (tabl. 15, et fig. 14B). Le deuxième facteur (21\% de la variance totale) est caractérisé par l'abondance des Enchytraeidae g. spp., de Cernosvitoviella $s p$., de Lumbricillus fennicus, des formes immatures du genre Lumbricillus et de Nais elinguis. II s'agit d'un facteur saisonnier, les espèces ou taxons cités étant plus abondants au printemps, fait bien connu pour $N$. elinguis (Lafont 1989).

Le troisième facteur $(13 \%$ de la variance totale) oppose $M$. argentea à $L$. fennicus et à $E$. tetraedra (tabl. 15). Dès le troisième facteur, l'analyse ne souligne que des points anecdotiques dans le présent jeu de données, points qui concernent des espèces moins fréquentes comme $M$. argentea. A l'aide d'une classification ascendante hiérarchique, prenant en considération les coordonnées factorielles des stations sur les trois premiers facteurs, on peut distinguer cinq groupes de relevés:

- le groupe G1 (fig. 14A) englobe les stations B68, C68, B29. Ces trois stations s'opposent à toutes les autres par leurs peuplements de Naididae;

- les groupes G2 et G3 comprennent les stations où dominent $P$. volki et Stylodrilus (B98, C98, C29, A05, A98, A29, C37). On notera que la station B98 ne renferme qu'un seul exemplaire du genre Stylodrilus (tabl. 14);

- Le groupe G4 est caractérisé par toutes les stations printanières de l'amont (mars 1987, A37, juin 1988, A68 et mai 1975, A55);

- Le groupe G5 renferme la seule station B37 (aval réchauffé en mars 1987 où les Enchytraeidae et $N$. elinguis dominent les peuplements).

Si l'on considère à présent l'évolution saisonnière de la station amont, de 1975 à 1987-89, on obtient la succession suivante à partir de la valeur moyenne des coordonnées factorielles sur F1-F2: juin 1988 (A68) 
Tableau 15. - Coefficients de corrélation des 16 espèces ou taxons avec les trois premiers facteurs ( $F 1, F 2, F 3$ ) de l'ACP.

Table 15. - Linear correlation coefficients of oligochaete species or taxa with the first three factors of the PCA (F1, F2, F3).

\begin{tabular}{|c|c|c|c|c|}
\hline $\begin{array}{l}\text { Pourcentage } \\
\text { Espèces }\end{array}$ & d'inertie & $\begin{array}{c}F 1 \\
31,7 \%\end{array}$ & $\begin{array}{r}F 2 \\
21 \%\end{array}$ & $\begin{array}{c}F 3 \\
13.4 \%\end{array}$ \\
\hline \multicolumn{5}{|l|}{ Immatures du genre } \\
\hline Stylodrilus & STOO & 0.83 & 0,05 & $-0,39$ \\
\hline Enchytraeidae & ENCH & 0.27 & $-0,72$ & 0,34 \\
\hline P. volki & PPVO & 0.78 & 0,21 & -0.32 \\
\hline 5. heringianus & STHE & 0.77 & 0.14 & $<-0.01$ \\
\hline N. elinguis & NAEL & $-0,26$ & $-0,75$ & $-0,13$ \\
\hline Cernosvitoviella sp. & CEOO & -0.43 & $-0,76$ & -0.15 \\
\hline L. fennicus & LCFE & 0.25 & $-0,62$ & 0,68 \\
\hline N. bretscher $i$ & NABR & $-0,86$ & $-0,10$ & -0.02 \\
\hline N. alpina & NAAL & -0.88 & 0,17 & $-0,91$ \\
\hline C. diaphanus & CHDI & -0.76 & 0,26 & 0,99 \\
\hline$\frac{\overline{N \text {. behningi }}}{\text { Immatures du genre }}$ & NABE & $-0,56$ & 0,52 & 0.23 \\
\hline Lumbricillus & LCOO & $-0,08$ & $-0,72$ & 0,13 \\
\hline M. argentea & MAAR & 0,09 & $-0,46$ & $-0,69$ \\
\hline E. tetraedra & EITE & 0.25 & 0,12 & 0.64 \\
\hline c. diastrorhus & CHDS & $-0,52$ & 0.12 & $-0,36$ \\
\hline N. pardalis & NAPA & -0.25 & $-0,36$ & $-0,37$ \\
\hline
\end{tabular}

$\rightarrow$ mars $1987 \quad$ (A37) $\rightarrow$ mai 1975 (A55) - février 1989 (A29) - septembre 1988 (A98) - août 1975 (A85) octobre 1975 (A05).

La succession est logique, avec une opposition printemps-automne quelle que soit l'année considérée. Seul le relevé $A 29$ fait exception à la règle, relevé où $M$. argentea (espèce décrivant des perturbations hydrauliques) présente ses effectifs les plus élevés dans le jeu de données.

Fig. 14. - Résullats de l'analyse en composantes principales :

A. carte factorielle des stations (plan F1-F2);

B. cercle des corrélations;

P.v. : P. volki, S.h.: S. heringianus, S.i. : immatures du genre Stylodrilus, L.f.: L. fennicus, L.i. : immatures du genre Lumbricillus, E.s. : Enchyiraeidae g. sp., N.a., N.b., N.e. : N. alpina, N. barbata, N. elinguis, C.d., C.s. : C. diaphanus, C. diastrophus.

Fig. 14. - Results of the PCA; $A=$ plot of sampling stations on the first plane; $B=$ correlation circle;

P.v. : P. volki, S.h. : S. heringianus, S.i. : immatures of the genus Stylodrilus, L.f. : L. fennicus, L.i. : immalures of the genus Lumbricillus, E.s.: Enchytraeidae g. sp., N.a., N.b., N.e.: N. alpina, $N$. barbala, N. elinguis, C.d., C.s. : $C$. diaphanus, $C$. diastrophus. 

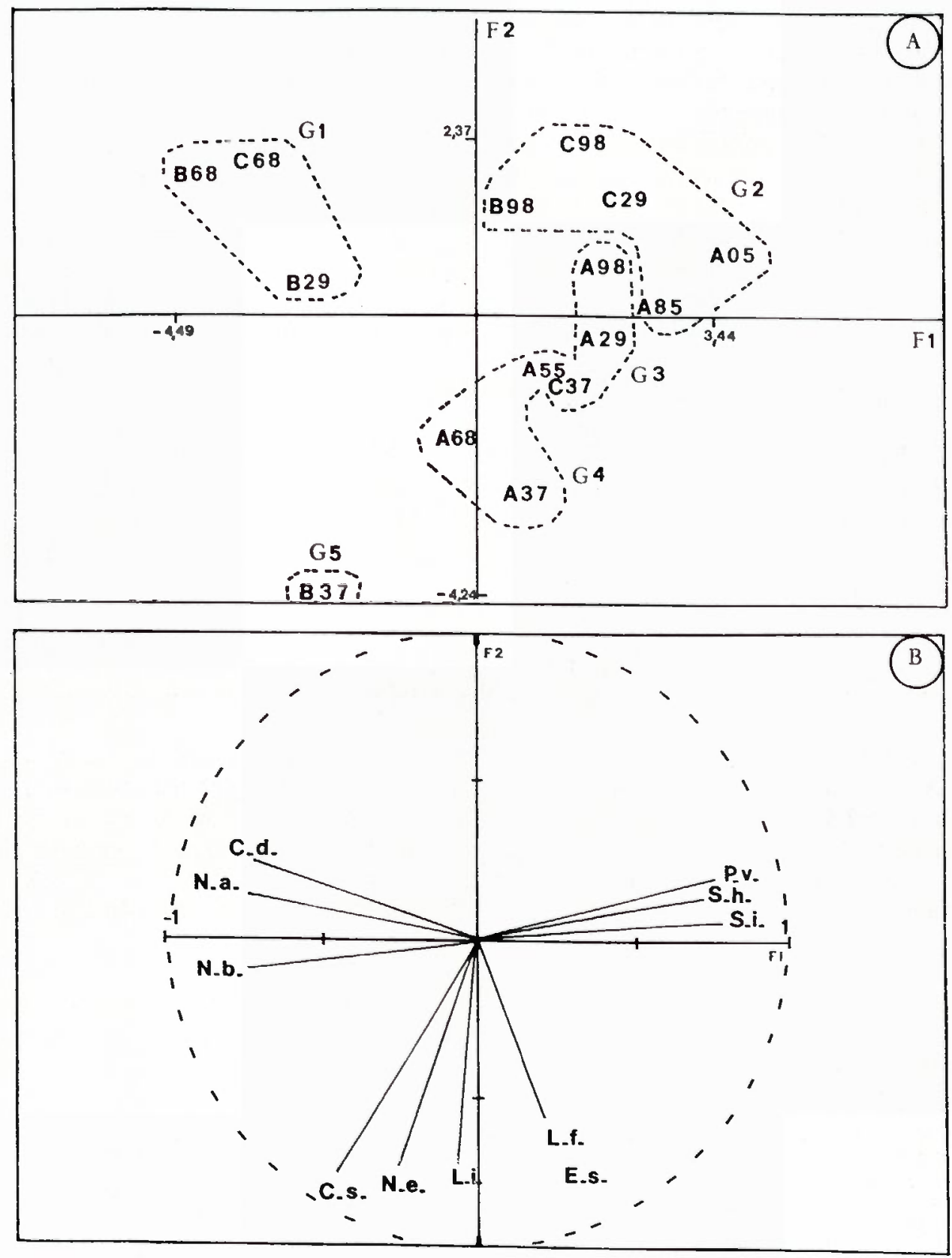
En conclusion, les peuplements de la station amont sont restés relativement stables dans le temps, en particulier ceux qui correspondent aux relevés printaniers (groupe G4, fig. 14A). Les principales différences concernent la présence significative en 1987-89 d'espèces caractéristiques d'échanges actifs nappe-cours d'eau, comme Cernosvitoviella sp. et $M$. argentea. On notera que la proportion de l'Enchytraeidae $P$. volki, autre espèce caractérisant de tels échanges, reste stable à 10 ans d'in- tervalle ( 0 à $62,1 \%$ en $1975,1,6$ à $71,7 \%$ en $1987-1989)$.

La présence des retenues artifjcielles à l'amont de Saint-Vulbas peut être tenue pour responsable de l'activation des échanges hydriques nappe-cours d'eau. L'impact de ces aménagements sur la densité des oligochètes n'est en revanche pas formellement prouvé. En effet, l'existence de rejets à caractère toxique (cf. chapitre 2) serait suffisante pour expliquer les diminutions d'effectifs.

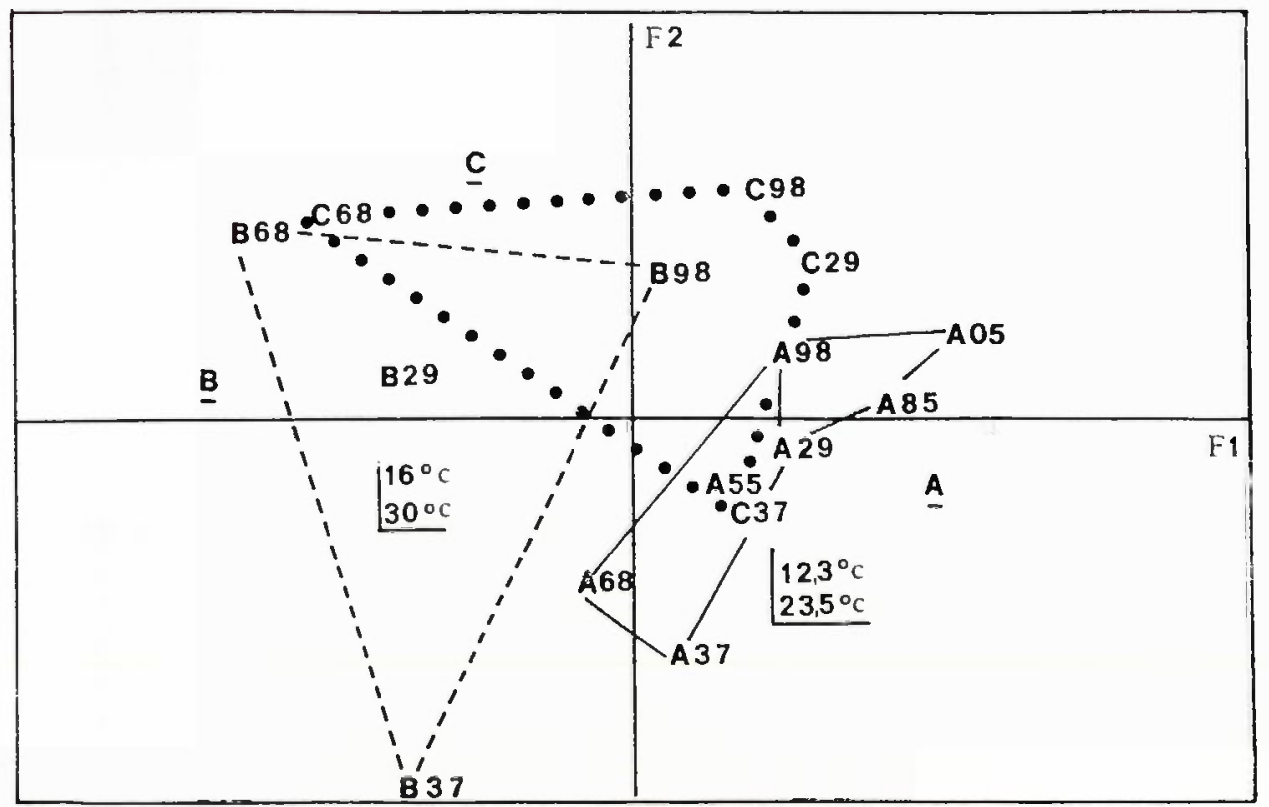

Fig. 15. - Polygones de dispersion des stations dans le plan F1-F2; en médaillon, températures moyennes et maximales relevées en 1988 sur les sites $A$ et $B$.

Fig. 15. - Plot of dispersion polygons of the sampling stations on the first plane of the PCA; for stations $A$ and $B$ mean and maximal water temperatures are noted. 
Quoiqu'il en soit, on dispose à présent d'une référence biologique bien établie pour aborder l'impact éventuel des rejets thermiques.

\subsubsection{Impact des rejets thermi- ques}

L'impact des rejets thermiques a été évalué à partir des campagnes où l'on disposait de relevés effectués simultanément sur les trois stations (campagnes de mars 1989, juin, août, septembre 1988, février 1989 , soit 12 relevés au total). Compte tenu de la nature des données, l'approche sera principalement descriptive.

Quatre observations semblent indiquer qu'il existe un impact significatif des rejets thermiques.

La première fait référence à l'ACP décrite dans le paragraphe précédent. En effet, les polygones de dispersion, obtenus en joignant la position extrême des relevés de chaque station sur le plan F1-F2 (fig. 15), permettent de séparer la station amont (polygone A) de la station soumise au réchauf-

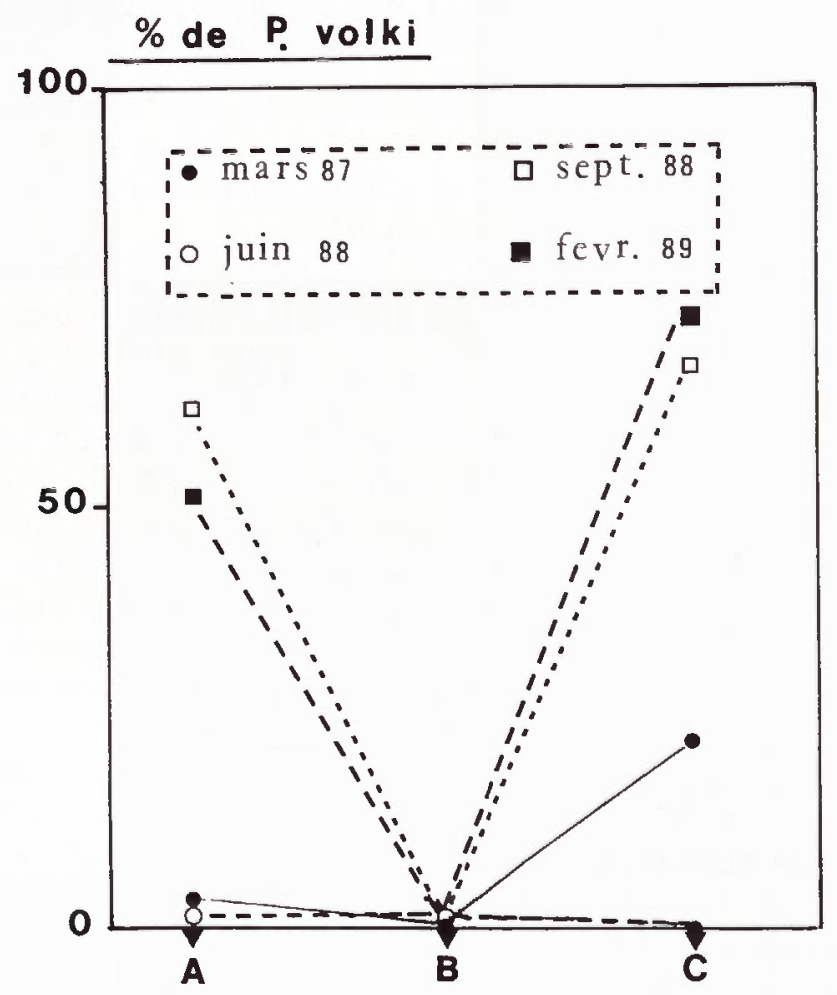

Fig. 16. - Variation du pourcentage de $P$. volki sur le site du Bugey.

Fig. 16. - Variation of the relative abundance of $P$. volki. 
fement (polygone B). Le polygone C (station $\mathrm{C}$, rive gauche moins affectée par les rejets d'eau chaude) se situe dans une position intermédiaire entre $A$ et $B$. La surface du polygone $B$ met en évidence les plus fortes variations saisonnières de l'abondance des oligochètes, variations que l'on peut également relier à un effet de la température (Nichols 1981).

La deuxième obsenvation concerne les effectifs et la position des espèces $P$. volki et $S$. heringianus (immatures + adultes) qui diminuent en général sur le secteur le plus chaud (station $B)$, le phénomène étant très significatif dans le cas de P. volki (tabl. 14 et fig. 16).

La troisième observation a été effectuée sur les effectifs moyens d'oligochètes de la station $B$ qui se révèlent inférieurs à ceux des autres stations $\left(A=122 / 0,1 \mathrm{~m}^{2}\right.$, $\left.C=112 / 0,1 \mathrm{~m}^{2}, \quad B=75 / 01 \mathrm{~m}^{2}\right)$. En particulier, ce secteur est pratiquement déserté par les oligochètes au mois d'août (2 exemplaires $/ 0,1 \mathrm{~m}^{2}$ ), alors que la température atteint $25^{\circ} \mathrm{C}$ en moyenne et $29,8^{\circ} \mathrm{C}$ au maximum (valeurs extrêmes de l'année 1988).

Enfin, le premier facteur de l'ACP et l'examen des données brutes (tabl. 14, fig. 14) oppose la station B (réchauffée) aux autres stations par un peuplement de Naididae en général plus abondant $(N$. elinguis, Nais bretscheri, Nais pardalis, Nais alpina). L'augmentation des effectifs du genre Nais a également été constatée par Nichols (1981) dans des eaux artificiellement réchauffées.

\subsubsection{Conclusion}

Les communautés d'oligochètes des sédiments grossiers présentent, à 10 années d'intervalle, une relative stabilité sur le site amont (Saint-Vulbas). Les variations observées semblent liées à une augmentation en 87-89 des échanges hydrodynamiques entre la nappe et le fleuve (impact des retenues), et probablement à une détérioration de la qualité du milieu liée à des rejets toxiques. L'instabilité des substrats grossiers, conséquence des variations brusques de niveau du fleuve, peut également être en partie responsable des changements observés.

L'impact des rejets d'eau chaude se superpose aux autres facteurs. Cet impact paraît se résumer de la façon suivante :

- disparition ou danger de disparition de certaines espèces (tabl. 15);

- baisse significative des effectifs d'oligochètes sur la station réchauffée, liée soit à un effet direct de la température, soit plus vraisemblablement à une synergie entre effet toxique et effet thermique (Dusoge \& Wisniewski 1976). Cette synergie peut en toute logique exister dans le cas d'un échauffement naturel et saisonnier des eaux;

- Naididae plus abondants et fortes variations saisonnières de l'abondance des peuplements d'oligochètes sur la station réchauffée.

Cependant, il convient de modérer ces affirmations. La température ne constitue pas le seul facteur d'altération des biocénoses comme nous l'avons vu. De plus, la station échauffée 
Tableau 16. - Données écologiques sur quelques espèces remarquables.

Table 16. - Ecological data on some noteworthy oligochaete species; impact of warmed waters.

\begin{tabular}{|c|c|}
\hline & COMMENTAIRES - CONCLUSIONS. \\
\hline Propappus volki & $\begin{array}{l}\text { Descripteur de l'intensite des echanges hydriques entre la nappe } \\
\text { et le cours d'eau (GASCHIGNARD-FOSSATI. 1986). } \\
\text { Sensible aux rejets industriels (LAFONT, 1989). } \\
\text { Thermophobe : absent de la station } 8 . \\
\text { En danger sur tout le site du Bugey (synergie temperature } \\
\text { toxiques). }\end{array}$ \\
\hline $\begin{array}{l}\text { Styojrilus heringianus } \\
\text { et immatures du genre } \\
\text { St.vlodrilus }\end{array}$ & $\begin{array}{l}\text { Sa prolifération peut être un signe d'instabilité des substrats } \\
\text { (Gaschignard. } 1984 \text {, a et b) : } \\
\text { Autres commentaires : cf. P. volki. }\end{array}$ \\
\hline $\begin{array}{l}\text { Nais.spp. (5 espèces. } \\
\text { sf. tab. 2) }\end{array}$ & $\begin{array}{l}\text { Peuplement qui devrait se maintenir sur la station réchauffee (B) } \\
\text { tant que les contaminations industrielles ne depasseront pas } \\
\text { le seuil actuel. } \\
\text { Peuplement thermophile (NICHOLS, 1981). }\end{array}$ \\
\hline $\begin{array}{l}\text { Cernosvitoviella sp. } \\
\text { Marionina argentea }\end{array}$ & $\begin{array}{l}\text { Descripteurs potentiels de l'intensite des échanges hydriques } \\
\text { entre la nappe et les eaux superficielles (LAFONT, 1989). } \\
\text { Peu abondants sur tout le site. sembleraient. pouvoir se maintenir } \\
\text { dans la station réchauffée. }\end{array}$ \\
\hline Eiseriella tetraedra & A disparu des trois stations (A. B et C) depuis 1975. \\
\hline $\begin{array}{l}\text { Lumbricillus fennicus et } \\
\text { immatures du genre } \\
\text { Lumbricillus }\end{array}$ & $\begin{array}{l}\text { Taxons plus abondants sur la station amont au printemps seulement, } \\
\text { rares ou absents sur tout le site aux autres saisons. } \\
\text { En danger du fait des contaminations toxiques et des élevations de } \\
\text { température, l'espèce } L \text {. fennicus. et les immatures qui } \\
\text { appartiennent vraisemblablement à cette espèce, se revélant } \\
\text { thermophobes y compris dans le secteur non réchauffe }(\mathrm{A}) \text {. Les } \\
\text { températures maximales ne doivent apparemment pas dépasser } 20^{\circ} \mathrm{C} \\
\text { environ. }\end{array}$ \\
\hline
\end{tabular}

se situe à proximité immédiate en rive droite de l'effluent de la centrale et les effets thermiques se révèlent bien moins accusés en rive gauche. On ajoutera enfin que le remplacement d'un peuplement à $P$. volki par un peuplement de Naididae constitue certes un effet significatif, mais on ne peut guère apprécier s'il s'agit d'un effet négatif ou positif. De plus, les Naididae ne s'avèrent pas très abondants (moins de 80 exemplaires $/ 0,1 \mathrm{~m}^{2}$ au maximum), ce qui enlève une certaine part de validité à l'observation. En revanche, la baisse des effectifs d'oligochètes constitue un impact objectivement négatif, mais l'élévation de la température n'est probablement pas la seule cause de cet impact, du moins dans la fourchette des valeurs actuelles. 


\section{4. - CONCLUSION GÉNÉRALE}

Le bilan général de l'étude se révèle certes complexe à établir. Toutefois, quelques grandes tendances se dégagent, que l'on peut résumer comme suit.

Au niveau de la station amont (Saint-Vulbas, A), on constate une baisse sensible de la qualité biologique par rapport à 1975 qui se traduit par une diminution de la richesse taxonomique des invertébrés (22 taxons ont disparu). Dans le cas précis des oligochètes, organismes moins mobiles que les insectes aquatiques, les effectifs baissent significativement entre 1975 et 1987-89, alors que la richesse spécifique et la nature des peuplements restent relativement stables.

Les causes de cette dégradation sont à rechercher dans le marnage important sur le secteur, lié à la gestion des barrages en amont, et vraisemblablement à divers rejets industriels. On notera que l'impact du marnage se traduit également par la prédominance d'espèces d'oligochètes décrivant une activation des échanges entre les eaux souterraines et superficielles.

Dans ce contexte, l'impact des rejets thermiques peut être mis en évidence, tout en soulignant que le réchauffement des eaux présente éventuellement un effet synergique avec celui des effluents industriels. Par exemple, un certain nombre d'observations propres à la station échauffée (tabl. 12 et 16), telles que la diminution de la richesse taxonomique des invertébrés, la baisse significative des effectifs d'oligochètes ou la disparition de certaines espèces (Potamanthus luteus, Propappus volki) constituent un impact négatif du réchauffement des eaux, allié probablement à celui des rejets industriels. En revanche, l'impact ne se révèle pas systématiquement négatif et traduit parfois des modifications de la structure des peuplements ou de la biologie des espèces (remplacement d'un peuplement à $P$. volki et $S$. heringianus par un peuplement à Naididae dans le cas des oligochètes, diapause écourtée chez les trichoptères...).

Dans le contexte général d'un impact global du marnage et du syndrome apparent de pollution industrielle sur tout le site du Bugey, les contraintes imposées au Centre de Production Nucléaire quant à l'échauffement des eaux semblent bien remplir leur rôle de limitation des impacts.

Les recommandations en matière de protection du site sont donc à première vue simples à préconiser :

- supprimer les rejets toxiques;

- maintenir les normes de l'échauffement actuellement fixées aux rejets de la Centrale.

En ce qui concerne l'impact des retenues de l'amont, le problème est plus délicat à résoudre et dépasse le seul cadre du site du Bugey.

Remerciements. Les auteurs remercient Madame M. Taillole qui a dactylographié le manuscrit. 


\section{BIBLIOGRAPHIE}

Agence de Bassin RMC Ministère de l'Environnement 1988. Qualité du fleuve Rhône. Synthèse des connaissances. 250 p. + annexes.

Arthur J.W., Zischke J.A. \& Ericksen G.L., 1982 : Effect of elevated water temperature on macro-invertebrate communities in outdoar experimental channels. Water Res. 16 : 1465-1477.

Bournaud M., Tachet H. \& Perrin J.F. 1982 : Les Hydropsychidae (Trichoptera) du haut-Rhône entre Genère et Lyon. Annls Limnol. 18(1): 61-80.

Bournaud M., Tachet H., Roux A.L. \& Auda Y. 1987a: The effects of seasonal and hydrological influences on the macroinvertebrates of the Rhone river, France. I. Méthodological aspects. Arch. Hydrobiol. 109 (2) : 287-304.

Boumaud M. Tachet H. \& Roux A.L. $1978 \mathrm{~b}$. The effects of seasonal and hydrological influences on the macroinvertebrates of the Rhône river, France. II. Ecological aspect. Arch. Hydrobiol./suppl. 76 (1/2): 25-51.

Cellot B. 1982 : Cycle annuel et zonation de la dérive des macroinvertébrés du Rhône en amont de Lyon. Thèse Doctorat $3^{\theta}$ cycle, Univ. Lyon I, 167 pages.

CTGREF 1977: Etude écologique du Rhône, sites de Bugey, Chavanoz et Tricastin, $102 \mathrm{p}$.

Degrange C. 1959 : Recherche sur la reproduction des Ephéméroptères. Trav. Lab. Hydrobio. et Piscic. Univ. Grenoble, 7-194.

Dessaix J. 1980: Les gammares du Rhône en amont de Lyon. Dynamique des populations et estimation de production. Thèse doct. spécialité. Univ. Lyon. $138 \mathrm{p}$.

Doledec S. 1987 : Etude des peuplements de macroinvertébrés benthiques de l'Ardèche dans son cours inférieur. Arch. Hydrobiol. 109 : 541-565.
Dusoge K. \& R. Wisniewski 1976 : Effect of heated waters on biocenosis of the moderately polluted Narew river. Macrobenthos. Pol. Arch. Hydrobiol., 23 (4) : 539-554.

El Kallab-Wakim A. 1978 : Etude du cycle biologique d'Heptagenia sulphurea Müller 1776) (Ephemeroptères, Heptageniidae) dans le Rhône à Lyon. Thèse doc. spéc. Univ. Lyon, $65 \mathrm{p}$.

Fey J.M. 1977 : Die Aufheitzung eines Mittelgebirgstlusses und ihre Aufwirkungen auf die zoozönose dargestelt and der Lenne (Sauerland). Arch. Hydrobiol./Suppl. 53, 3 : 307-363.

Fruget J.F. 1989 : L'aménagement du Bas-Rhône. Evolution du fleuve et influence sur les peuplements de macroinvertébrés benthiques. Thèse de Doctorat, UCBL Lyon I: $481 \mathrm{p}$.

Gaschignard O. 1984a : La granulométrie du sédiment et l'évolution du benthos dans un bras vif du Rhône. Rev. Sciences de l'Eau, 3 : 279-293.

Gaschignard O. 1984b : Impact d'une crue sur les macroinvertébrés benthiques d'un bras du Rhône. Verh. Internat. Verein. Limnol., 22 : 1997-2001.

Gaschignard-Fossati O., \& Berly A. 1987 : Impact of large discharge fluctuations on the macroinvertebrate populations downstream of a dam. J.F. Crang and J.P. Kemper (eds). 3rd Int. Symp. on Regulated stream Edmonton 1985: 145-161.

Gaschignard-Fossati O. 1986 : Répartition spatiale des macroinvertébrés benthiques d'un bras vif du Rhône; rôle des crues et dynamique saisonnière. Thèse de Doctorat, Université Lyon I: 197 p. + annexes.

Hauer F.R. \& Stanford J.A. 1982 : Ecological responses of Hydropsychid caddisflies to stream regulation. Can J. Fish. Sci. 39 : 1235-1242.

Hynes M.B.H. 1955 : The reproduction cycle of some british freshwater gammaridae. J. Anim. Ecol. 24 : 352-387. 
Iversen T.M. \& Jessen J. 1977 : Life cycle drift and production of Gammarus pulex L. (Amphipoda) in a Danish spring. Freshwater biol., 7 : 287-296.

Lafont M., 1989 : Contribution à la gestion des eaux continentales : utilisation des oligochètes comme descripteurs de l'état biologique et du degré de pollution des eaux et des sédiments. Thèse de Doctorat d'Etat ès Sciences, UCBL Lyon I: 311 p. + annexes $92 \mathrm{p}$.

Lafont M. \& Durbec A. 1990a : Description biologique des relations hydrauliques entre la nappe et les eaux superficielles: Application à l'impact de gravières. Actes du Symposium "Bravo l'Eau", Colmar 28-30 mars 1990: 11 p.

Lafont M. \& Durbec A. 1990b : Essai de description biologique des interactions entre eau de surface et eau souterraine: Application à l'évaluation de la vulnérabilité d'un aquifère à la pollution d'un fleuve. Annls. Limmol. : 26 (2-3) : 119-129.

Langford T.E. 1972 : A comparative assessment of thermal effects in some British and North American rivers : 319351. In River Ecology and mass. Ogleslug R.T. Carison C.A. Mc Cann J.A. Academic Press $n^{\circ} 9$ London.

Mouthon J. 1981 : Les mollusques et la pollution des eaux douces: ébauche d'une gamme de polluosensibilité des espèces. Bijdragen Tot. de Dierkinde, 51 (2) : 250-258.

Mouvet C., Couturieux N., Morhain E. 1986 : Contamination métallique et organochlorée des mousses aquatiques du Rhône dans le secteur de l'Usine TREDI. Rapport Service de la Navigation Rhône-Saône, 9 p.

Munn M.D. \& Brusven M.A. 1987 : Discontinuity of Trichopteran (Caddisfly) communities in Regulated waters of the cleanwater river, Idaho U.S.A. Regulated rivers : 61-69.

Nichols S.J. 1981 : Effect of thermal effluents on oligochaetes in Keowee reservoir, South Carolina. Hydrobiologia, $79: 129-136$.
Nisbet M. \& Verneaux J. 1970 : Composantes chimiques des eaux courantes et propositions de classes en tant que bases d'interprétation des analyses physico-chimiques. Annls. Limnol. $6(2): 161190$.

Obrdlik P., Adamek Z. \& Zahradka J. 1979 : Mayfly fauna (Ephemeroptera) and the biology of the species Potamanthus luteus (L.) in a warmed stretch of the Oslava river. Hydrobiologia, 67, 2 : 129-140.

Perrin J.F. 1978 : Signification écologique des peuplements benthiques du HautRhône français. Thèse Doct. Spécialité. Univ. Lyon, $171 \mathrm{p}$.

Perrin J.F. \& Roux A.L. 1978. La macrofaune benthique du Rhône. Verh. intem. Verein. Limnol. 20 : 1494-1502.

Roger M.C. \& B. Faessel 1989 : Effet de l'échauffement artificiel de l'eau du Rhône sur le développement et la production des Gammaridae. Hydroécol. Appl., 1/2 : 53-83.

Roux A.L. 1970 : Le cycle de reproduction de deux espèces étroitement parentes de crustacés Amphipodes : Gammarus pulex et Gammarus fossarum. Ann/s Limnol. 6 (1) : 27-49.

Roux A.L., Tachet H., Neyron M. 1976 : Une technique simple et peu onéreuse pour l'étude des macroinvertébrés benthiques des grands fleuves. Bull. Ecol. 7 (4) : 493-496.

Scullion J., Parish C.A., Morgan N. \& Edwarde R.W. 1982 : Comparison of bentic macroinvertebrate fauna and substratum composition in ripples and pools in the impounded River Elan and the irregulated river Wye, Mid-Wales. Freshwat. biol. 12 : 579-595.

Scullion J. \& Sinton A. 1983 : Effects of artificial freshets on substratum composition, benthic invertebrate fauna and invertebrate drift in two impounded rivers in Mid-Wales. Hydrobiologia 107 : 261-269.

Tachet H. \& Bournaud M. 1981 : Cycles biologiques des Hydropsychidae et 
d'un Polycentropodidae (Trichoptera) dans le Rhône en amont de Lyon. In Proc. of the 3rd Int. Sympos. on Trichoptera ed G.P. Moretti: 347-364, Junk, The Hague.

Talmage S.S. \& Coutant C.C. 1980 : Thermal effects. Rewies and Models. Joum. WPCF 52 (6) : 1575-1616.

Vaillant F. 1962 : Un Empididae destructeur de Simulies. Bull. Soc. Zool. Fr. T. $77: 371-379$.

Vannote R.L. \& Sweeny B.W. 1980 : Geographic analysis of thermal equilibra: A conceptual model for evaluating the effect of natural and modified thermal regimes on aquatic insect communities. Am. Wati. 115 : 667-695.

Verneaux J. 1973: Cours d'eau de Franche-Comté (Massif du Jura). Recherche écologique sur le réseau hydrographique du Doubs. Essai de biotypologie. Thèse Doct. Sci. Univ. Besançon, $260 \mathrm{p}$.

Verneaux J., Faessel B. \& Malesieux G. 1976 Note préliminaire à la proposition de nouvelles méthodes de détermination de la qualité des eaux courantes. Travaux Div. Qual. Eaux P. et Pisc. Ronéo $14 \mathrm{p}$.

Ward J.V. \& Stanford J.A. 1979 : Ecological factors controlling stream zoobenthos with emphasis on thermal modification of regulated streams : 35 55. In The ecology of regulated streams Ward and Stanford edit. Plenum Press. N.Y. 385 p.

Williams G.P. \& Wolman G. 1984 : Downstreams effects of dams an alluvial rivers. Geological survey professional paper, $83 \mathrm{p}$. 